\title{
Recognizing Cluster Algebras of Finite type
}

\author{
Ahmet I. Seven* \\ Northeastern University, Boston, MA 02115, USA and \\ Middle East Technical University, Ankara, 06531, Turkey \\ aseven@metu.edu.tr
}

Submitted: Jul 25, 2004; Accepted: Dec 5, 2006; Published: Jan 3, 2007

Mathematics Subject Classifications: 05E99

\begin{abstract}
We compute the list of all minimal 2-infinite diagrams, which are cluster algebraic analogues of extended Dynkin graphs.
\end{abstract}

\section{Introduction}

Cluster algebras were introduced in [4] by Fomin and Zelevinsky to provide an algebraic framework for the study of canonical bases in quantum groups. Since their introduction, it has also been observed that cluster algebras are closely related with different areas in mathematics. For example; they provide a natural algebraic set-up to study recursively defined rational functions in combinatorics and number theory [3]. In geometry, they introduce natural Poisson transformations [6]. In representation theory, they form a natural algebraic framework to study positivity [1].

One of the most striking results in the theory of cluster algebras due to S. Fomin and A. Zelevinsky is the classification of cluster algebras of finite type, which turns out to be identical to the Cartan-Killing classification [4]. This result can be stated purely combinatorially in terms of certain transformations, called mutations, on certain graphs. To be more precise, let us assume that $\Gamma$ is a finite directed graph whose edges are weighted with positive integers. We call $\Gamma$ a diagram if it has the following property: the product of weights along any cycle is a perfect square, i.e. the square of an integer. For any vertex $k$ in $\Gamma$, the mutation $\mu_{k}$ in the direction $k$ is the transformation that changes $\Gamma$ as follows:

- The orientations of all edges incident to $k$ are reversed, their weights intact.

\footnotetext{
*The author's research was supported in part by Andrei Zelevinsky's NSF grant \#DMS-0200299.
} 
- For any vertices $i$ and $j$ which are connected in $\Gamma$ via a two-edge oriented path going through $k$ (refer to Figure 1 for the rest of notation), the direction of the edge $(i, j)$ in $\mu_{k}(\Gamma)$ and its weight $c^{\prime}$ are uniquely determined by the rule

$$
\pm \sqrt{c} \pm \sqrt{c^{\prime}}=\sqrt{a b}
$$

where the sign before $\sqrt{c}$ (resp., before $\sqrt{c^{\prime}}$ ) is " + " if $i, j, k$ form an oriented cycle in $\Gamma$ (resp., in $\mu_{k}(\Gamma)$ ), and is "-" otherwise. Here either $c$ or $c^{\prime}$ can be equal to 0 , which means that the corresponding edge is absent.

- The rest of the edges and their weights in $\Gamma$ remain unchanged.

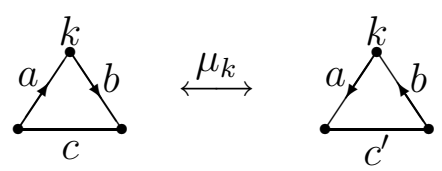

Figure 1: Diagram mutation

It is not hard to show that the resulting weighted graph is a diagram; in particular, its edge-weights are positive integers. It is also easy to check that $\mu_{k}$ is involutive, i.e. $\mu_{k}^{2}(\Gamma)=\Gamma$. Two diagrams $\Gamma$ and $\Gamma^{\prime}$ related by a sequence of diagram mutations are called mutation equivalent. A diagram is called $2-$ finite if every mutation equivalent diagram has all edge weights equal to 1,2 or 3 . The combinatorial part of the classification theorem in [5] is the following: a diagram is 2-finite if and only if it is mutation equivalent to a Dynkin diagram, i.e. a diagram whose underlying undirected graph is a Dynkin graph. However, in [5], an algorithm for checking whether a given diagram is mutation equivalent to a Dynkin diagram is not given. In particular, we do not know how many mutations one needs to perform to show that a given diagram is mutation equivalent to a particular Dynkin diagram, say, $E_{8}$. This makes the following recognition problem natural:

Problem 1.1 Recognition Problem for 2-finite diagrams: How to recognize whether a given diagram $\Gamma$ is 2-finite without having to perform an unspecified number of mutations.

In this paper, we solve Problem 1.1 completely by providing the list of all minimal 2-infinite diagrams (Section 8). The list contains all extended Dynkin diagrams but also has 6 more infinite series, and a substantial number of exceptional diagrams with at most 9 vertices. For the proof of this fact, we first show that any diagram in our list is minimal 2 -infinite. To prove that any minimal 2-infinite diagram is indeed in our list, we use an inductive argument. The basis of the induction is the following fact: our list contains any two-vertex diagram with the edge weight greater than or equal to 4 . The inductive step is the following statement: if a diagram $\Gamma$ contains a subdiagram that belongs to our list, then, for any vertex $k$ in $\Gamma$, the diagram $\mu_{k}(\Gamma)$ also contains a subdiagram from our list (Lemmas 3.4 and 3.5). Those two properties imply that our list contains all minimal 2-infinite diagrams. To be more precise, let us assume that $\Gamma$ is a minimal 2-infinite 
diagram. Then, by definition, there is a sequence of mutations $\mu_{r}, \ldots, \mu_{1}$ such that the diagram $\Gamma^{\prime}=\mu_{r} \circ \ldots \circ \mu_{1}(\Gamma)$ contains an edge whose weight is greater than or equal to 4. Here we note that $\Gamma=\mu_{1} \circ \ldots \circ \mu_{r}\left(\Gamma^{\prime}\right)$ because mutations are involutive. Thus, by induction on $k$, the diagram $\Gamma$ contains a subdiagram $\Gamma^{\prime}$ from our list. Since $\Gamma$ is minimal 2-infinite we have $\Gamma=\Gamma^{\prime}$, showing that our list is a complete list of minimal 2-infinite diagrams. We give a more detailed outline of this proof in Section 3.

We have used some computer assistance to produce our list of diagrams and prove that it really is the list of minimal 2-infinite diagrams. More specifically, we use computer to obtain exceptional minimal 2-infinite diagrams, which are the minimal 2-infinite diagrams that do not appear in series. We first used a theoretical argument to show that those exceptional diagrams can have at most 9 vertices, even so it presented a challenge for us to compute them explicitly because we needed, in one form or another, a fast method to check using a computer if a given diagram is 2-finite. In Section 5, we develop such a method for simply-laced diagrams, here a diagram is called simply-laced if all of its edges have weight equal to 1 . The basic idea of our method is to view the underlying graph of a diagram as an alternating bilinear form on a vector space over the 2-element field, and describe an arbitrary simply-laced 2-finite diagram using algebraic invariants of the corresponding bilinear form ${ }^{1}$. A nice combinatorial set-up to carry out this idea is provided by a class of (undirected) graph transformations called basic moves, which were introduced and studied in $[2,11]$. A basic move is a simpler operation than a mutation; there is also a classification of graphs under basic moves using algebraic and combinatorial invariants which can be easily implemented $[9,11]$. We take advantage of this classification thanks to our following characterization: a simply-laced diagram that does not contain any non-oriented cycle is 2-finite if and only if its underlying graph can be obtained from a Dynkin graph using basic moves (Theorem 5.3). Using this description, we design and implement the algorithm in Section 5.4, computing the exceptional minimal 2-infinite diagrams. Our computer program is available at [15].

In addition to giving an explicit description of minimal 2-infinite diagrams, we determine representatives for their mutation classes. In particular, we prove that any minimal 2-infinite diagram with at least 5 vertices is mutation equivalent to an extended Dynkin diagram (Theorem 3.2). We also remark that one can enlarge the set of extended Dynkin diagrams by including some other representatives giving the following "intermediate" recognition criterion: a diagram is 2 -infinite if and only, using at most 9 mutations, it can be transformed into a diagram which contains one of the distinguished representatives (Remark 7.1).

Another long list of directed graphs (quivers) was obtained by Happel and Vossieck in [8] to classify finite dimensional algebras which are of minimal infinite representation type. We observed that our list of simply-laced minimal 2-infinite diagrams is the same as the list of Happel and Vossieck up to a natural operation of replacing the dotted edges indicating relations of quivers in [8] by arrows in the reverse direction. This remarkable coincidence of

\footnotetext{
${ }^{1}$ After the first version of this paper appeared, M. Barot, C. Geiss and A. Zelevinsky had the paper "Cluster algebras of finite type and positive symmetrizable matrices" (J. London Math. Soc. (2006) no:3, 545-564), where they obtained a description of 2-finite diagrams using bilinear forms over integers.
} 
such long lists suggests a close relation between the associated finite dimensional algebras and cluster algebras, which we will explore in a separate publication. Let us also note that our list contains non-simply-laced diagrams while the diagrams in [8] are simply-laced.

The paper is organized as follows. In Section 2, we give basic definitions. In Section 3, we state our main results and outline their proofs. In Section 4, we prove some statements that allow us to compute series of minimal 2-infinite diagrams. In Section 5, we compute exceptional simply-laced minimal 2-infinite diagrams using basic moves. In Sections 6 and 7 , we prove our main results. Section 8 is our list of minimal 2-infinite diagrams.

\section{Basic Definitions}

In this section, we recall some definitions and statements from $[4,5]$. We start with the skew-symmetrizability property of an integer matrix [4, Definition 4.4].

Definition 2.1 Let $B$ be a $n \times n$ matrix whose entries are integers. The matrix $B$ is called skew-symmetrizable if there exists a diagonal matrix $D$ with positive diagonal entries such that $D B$ is skew-symmetric.

For any skew-symmetrizable matrix $B$, Fomin and Zelevinsky introduced a weighted directed graph as follows ([5, Definition 7.3]).

Definition 2.2 Let $n$ be a positive integer and let $I=\{1,2, \ldots, n\}$. The diagram of a skew-symmetrizable integer matrix $B=\left(b_{i j}\right)_{i, j \in I}$ is the weighted directed graph $\Gamma(B)$ with the vertex set $I$ such that there is a directed edge from $i$ to $j$ if and only if $b_{i j}>0$, and this edge is assigned the weight $\left|b_{i j} b_{j i}\right|$.

According to [5, Lemma 7.4]; if $B$ is a skew-symmetrizable matrix, then, for all $k \geq 3$ and all $i_{1}, \ldots, i_{k}$, it satisfies

$$
b_{i_{1} i_{2}} b_{i_{2} i_{3}} \cdots b_{i_{k} i_{1}}=(-1)^{k} b_{i_{2} i_{1}} b_{i_{3} i_{2}} \cdots b_{i_{1} i_{k}}
$$

In particular, if the edges $e_{1}, e_{2}, \ldots, e_{r}$ with weights $w_{1}, w_{2}, \ldots, w_{r}$ form an induced cycle (which is not necessarily oriented) in $\Gamma(B)$, then the product $w_{1} w_{2} \ldots w_{r}$ is a perfect square. Thus we can naturally define a diagram as follows:

Definition 2.3 A diagram $\Gamma$ is a finite directed graph whose edges are weighted with positive integers such that the product of weights along any cycle is a perfect square.

By some abuse of notation, we denote by the same symbol $\Gamma$ the underlying undirected graph of a diagram. If an edge $e=[i, j]$ has weight equal to 1 , then we call e weightless and do not specify its weight in the picture. If all the edges are weightless, then we call $\Gamma$ simply-laced. By a subdiagram of $\Gamma$, we always mean a diagram $\Gamma^{\prime}$ obtained from $\Gamma$ by taking an induced directed subgraph on a subset of vertices and keeping all its edge weights the same as in $\Gamma\left[5\right.$, Definition 9.1]. We will denote this by $\Gamma^{\prime} \subset \Gamma$. 
For any vertex $k$ in a diagram $\Gamma$, there is the associated mutation $\mu_{k}$ which changes $\Gamma$ as described in Fig. 1. This operation naturally defines an equivalence relation on the set of all diagrams. More precisely, two diagrams are called mutation equivalent if they can be obtained from each other by applying a sequence of mutations. An important type of diagrams that behave very nicely under mutations are 2-finite diagrams:

Definition 2.4 A diagram $\Gamma$ is called 2-finite if any diagram $\Gamma^{\prime}$ which is mutation equivalent to $\Gamma$ has all edge weights equal to 1,2 or 3 . A diagram is called 2 -infinite if it is not 2-finite.

Let us note that a subdiagram of a 2-finite diagram is 2-finite. We also note that there are only finitely many diagrams which are mutation equivalent to a given 2-finite diagram.

2-finite diagrams were classified by Fomin and Zelevinsky in [5]. Their classification is identical to the Cartan-Killing classification. More precisely:

Theorem 2.5 A diagram is 2-finite if and only if it is mutation equivalent to an arbitrarily oriented Dynkin diagram (Fig. 2).

It is a natural problem to give an explicit description of 2-finite diagrams (Problem 1.1). A conceptual solution to this problem could be obtained by finding the list of all minimal 2-infinite diagrams. More precisely:

Definition 2.6 A diagram $\Gamma$ is called minimal 2-infinite if it is 2-infinite and any proper subdiagram of $\Gamma$ is 2-finite.

Clearly one has the following:

a diagram $\Gamma$ is 2 -infinite if and only if it contains a subdiagram which is minimal 2-infinite.

In Section 8, we give a complete list of minimal 2-infinite diagrams, solving Problem 1.1.

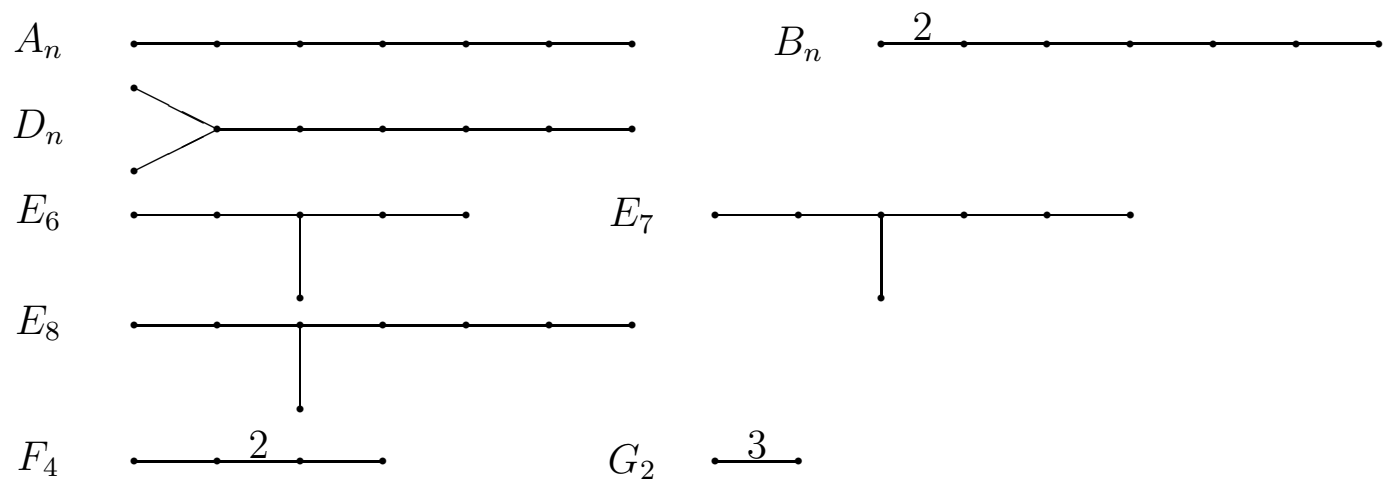

Figure 2: Dynkin diagrams 


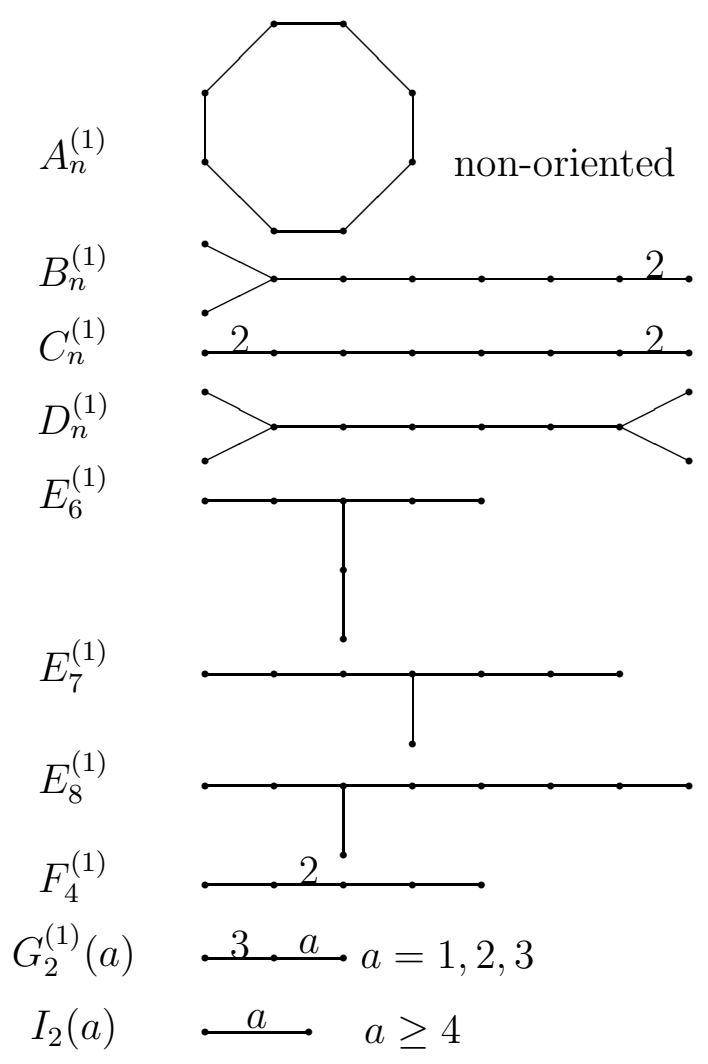

Figure 3: Extended Dynkin diagrams

\section{Main Results}

Throughout the paper, we assume that all diagrams are connected. We also assume, unless otherwise stated, that any diagram has an arbitrary orientation which does not contain any non-oriented cycle.

Our main result is the following statement:

Theorem 3.1 The list of minimal D-infinite diagrams consists precisely of the diagrams given in Section 8.

We also determine representatives for mutation classes of minimal 2-infinite diagrams as follows:

Theorem 3.2 Any minimal 2-infinite diagram is either one of the diagrams in Table 2 (Section 8) or it is mutation equivalent to an extended Dynkin diagram (Fig. 3).

We prove Theorem 3.1 as follows. We first show that any diagram in our list is minimal 2-infinite: 
Lemma 3.3 Any diagram $\Gamma$ in Section 8 is minimal 2-infinite.

Next, to complete the proof of Theorem 3.1, we show that any minimal 2-infinite diagram is indeed one of the diagrams in Section 8. For this, we recall that any 2-infinite diagram, in particular any minimal one, is mutation equivalent to a diagram which contains a subdiagram of the form $I_{2}(a), a \geq 4$ :

$$
\stackrel{a}{\longrightarrow} a \geq 4
$$

To be more precise, let us assume that $X^{\prime}$ is a 2 -infinite diagram and $\mu_{r}, \ldots, \mu_{1}$ a sequence of mutations such that the diagram $X=\mu_{r} \circ \ldots \circ \mu_{1}\left(X^{\prime}\right)$ contains a subdiagram of the form $I_{2}(a), a \geq 4$. Here we note that $X^{\prime}=\mu_{1} \circ \ldots \circ \mu_{r}(X)$ because mutations are involutive. We prove, by induction on $r$, that $X^{\prime}$ contains a subdiagram from our list, so if $X^{\prime}$ is minimal 2-infinite then this subdiagram must be $X^{\prime}$ itself (because any diagram in our list is 2-infinite), proving Theorem 3.1. The basis of the induction is the fact that any diagram of the form $I_{2}(a), a \geq 4$ is included in Section 8 (Table 1). The inductive step is the following statement:

If a diagram $X$ contains a subdiagram $\Gamma$ from Section 8, then, for any vertex $k$ in $X$, the diagram $\mu_{k}(X)$ also contains a subdiagram from Section 8 .

To establish (3.1), we consider it in two possible cases: the vertex $k$ being contained in $\Gamma$ or not. If $k$ is a vertex of $\Gamma$, we show that $\mu_{k}(\Gamma)$ contains a subdiagram from our list. If $k$ is not in $\Gamma$, we denote by $\Gamma k$ the minimal subdiagram of $X$ that contains $\Gamma$ and $k$, and show that $\mu_{k}(\Gamma k)$ contains a subdiagram from our list. For this we will assume, without loss of generality, that $k$ is connected to (at least two vertices in) $\Gamma$ because otherwise $\mu_{k}$ does not effect $\Gamma$ (Fig 1). In short, we prove the following two statements to show that (3.1) is satisfied:

Lemma 3.4 Let $\Gamma$ be an arbitrary diagram in Section 8. If $k$ is a vertex in $\Gamma$, then $\mu_{k}(\Gamma)$ contains a subdiagram $\Gamma^{\prime}$ which is in Section 8. Furthermore, if $\Gamma$ is in Table 1, then $\Gamma^{\prime}$ can be chosen from Table 1 .

Lemma 3.5 Suppose that $\Gamma$ is an arbitrary diagram in Section 8. Let $\Gamma k$ be a diagram obtained from $\Gamma$ by adjoining a vertex $k$. Then $\mu_{k}(\Gamma k)$ contains a subdiagram $\Gamma^{\prime}$ which is in Section 8.

Let us note that Lemmas 3.3, 3.4, 3.5 prove Theorem 3.1. We prove those lemmas in Sections 6.1, 6.2 and 7. The proof of Lemma 3.5 is more involved, therefore, for the convenience of the reader, here we discuss an outline of our proof in some detail. To prove this lemma we assume, without loss of generality, that $\Gamma k$ does not have any subdiagram which contains $k$ and belongs to our list (otherwise Lemma 3.4 applies). This assumption greatly restricts possible non-simply-laced $\Gamma k$ and we manage to obtain the lemma for such $\Gamma k$ using a case-by-case analysis. To treat simply-laced $\Gamma k$, it turns out to be convenient for us to consider them in two classes: those that do not contain any 
subdiagram which is mutation equivalent to the Dynkin diagram $E_{6}$ and those that do. If a simply-laced $\Gamma k$ belongs to the first class, then $\Gamma$ is in Table 1 (because any simply-laced diagram in other tables contains a subdiagram which is mutation equivalent to $E_{6}$ ). For such $\Gamma k$, we obtain the lemma from the following stronger statement:

Proposition 3.6 Suppose that $\Gamma$ is a simply-laced diagram in Table 1 (Section 8), i.e. $\Gamma$ is one of the following diagrams: $A_{n}^{(1)}, D_{n}^{(1)}, D_{n}^{(1)}(m, r), D_{n}^{(1)}(r), D_{n}^{(1)}(m, r, s)$. Let $\Gamma k$ be a simply-laced diagram obtained from $\Gamma$ by adjoining a vertex $k$. Suppose that $k$ is connected to at least two vertices in $\Gamma$. Suppose also that

the vertex $k$ is not contained in any subdiagram $E \subset \Gamma k$ such that $E$ is mutation equivalent to $E_{6}$.

Then (precisely) one of the following holds:

$k$ is contained in a diagram $\Gamma^{\prime \prime} \subset \Gamma k$ such that $\Gamma^{\prime \prime}$ is in Table 1, the diagram $\mu_{k}(\Gamma k)$ is in Table 1.

Let us note that if (3.3) is satisfied, then Lemma 3.4 applies, giving the same conclusion as Lemma 3.5. Now to complete the proof of Lemma 3.5, we need to establish it for $\Gamma k$ that contains a subdiagram, say $E$, which is mutation equivalent to $E_{6}$. For this we first show that $\mu_{k}(\Gamma k)$ contains a minimal 2-infinite diagram which has at most 9 vertices, then we show that any such minimal 2-infinite diagram is contained in our list. The first part is obvious if $\Gamma k$, thus $\mu_{k}(\Gamma k)$, has at most 9 vertices (recall that $\Gamma k$ is 2 -infinite, so $\mu_{k}(\Gamma k)$ is also 2-infinite thus contains a minimal 2-infinite diagram). For larger $\Gamma k$, we first observe the following fact in Corollary 5.6: any (simply-laced) diagram which has at least 9 vertices and contains a subdiagram mutation equivalent to $E_{6}$ is 2 -infinite. To use this fact in our set-up, we also observe that if $\Gamma k$ has at least 10 vertices then there exists a connected subdiagram $X k \subset \Gamma k$ of 9 vertices which contains both $E$ and $k$ (Section 7.1). Then $X k$ must be 2-infinite by the mentioned fact, so it contains a minimal 2 -infinite subdiagram, say $M$, which has at most 9 vertices. We note that $M$ contains $k$ because any subdiagram of $X k$ that does not contain $k$ is a proper subdiagram of $\Gamma$, and any proper subdiagram of $\Gamma$ is 2-finite because $\Gamma$ is minimal 2-infinite. Let us also note that $\mu_{k}(M)$, which is a subdiagram of $\mu_{k}(\Gamma k)$, is also 2-infinite, so it contains a minimal 2 -infinite diagram which has at most 9 vertices. Thus, to complete the proof of Lemma 3.5, it is enough to show that any minimal 2-infinite diagram $M$ with at most 9 vertices is contained in Section 8:

Proposition 3.7 Any simply-laced minimal 2-infinite diagram which has at most 9 vertices is contained in Section 8.

We prove the proposition using some theory that we develop in Section 5 along with some computer assistance. To motivate for our proof, let us first note that we may obtain 
all simply-laced minimal 2-infinite diagrams (with at most 9 vertices) as follows: first we compute all simply-laced 2-finite diagrams (with at most 8 vertices) mutating the corresponding Dynkin diagrams, then extend any 2-finite diagram by connecting, in all possible ways, an additional vertex such that the resulting diagram is 2 -infinite and any proper subdiagram is 2-finite. To implement the second step of this algorithm, we need an efficient method to check, possibly using a computer, if a given simply-laced diagram is 2-finite. Our basic idea to develop such a method is to view the underlying graph of a diagram as an alternating bilinear form on a vector space over the 2-element field, and characterize an arbitrary (simply-laced) 2-finite diagram using algebraic invariants of the corresponding bilinear form. A nice combinatorial set-up to carry out this idea is provided by a class of (undirected) graph transformations called basic moves, which were introduced and studied in $[2,11]$. A basic move changes a graph as follows: it introduces or deletes edges containing a fixed vertex connected to a given vertex (thus a basic move is assigned to a pair of vertices connected to each other, for a precise description see Definition 5.1). We note in Proposition 5.2 that the underlying graphs of mutation-equivalent simplylaced diagrams can be obtained from each other by a sequence of basic moves. We prove the converse of this statement for 2-finite diagrams: any simply-laced diagram that does not contain any non-oriented cycle is 2-finite if and only if its underlying graph can be obtained from a Dynkin graph using basic moves (Theorem 5.3). The advantage of characterizing 2-finite diagrams using basic moves is that a basic move is a simpler operation than a mutation; there is also a classification of graphs under basic moves using algebraic and combinatorial invariants which can be easily implemented $[9,11]$. In Proposition 5.7, we give such a characterization for graphs that can be obtained from Dynkin graphs with 6,7 or 8 vertices using basic moves. Using this description, we design and implement the algorithm in Section 5.4, obtaining all simply-laced minimal 2-infinite diagrams that contain a subdiagram which is mutation equivalent to $E_{6}$ (our computer program is available at [15]). For the remaining simply-laced minimal 2-infinite diagrams, we prove that they must belong to Table 1 (Corollary 5.14), completing the proof of Proposition 3.7.

We will prove Theorem 3.1 in Section 7. We prove Theorem 3.2 in Section 7.4. The reader may note from the outline in this section that we use most of our results to show Lemma 3.5 for a simply-laced diagram $\Gamma$ in our list. As we mentioned, we also show the lemma for a non-simply-laced diagram in our list. For such diagrams, we show the lemma directly, without referring to any non-trivial statements; this is because those diagrams are fairly simple (Table 1-3). In the course of the proof, we use same type of arguments repeatedly. Since we also need to save space, in this paper we do not include our treatment of all non-simply-laced diagrams; we do a representative one in Section 7.3. For the complete proof, we refer the reader to the long version of this paper which is available at [12] or refer to [13]. There is also a similar type of referring in Section 4.2. 


\section{Series of minimal 2-infinite diagrams}

In this section we prove Proposition 3.6. For this it will be convenient for us to prove first a slightly stronger statement for the diagram $A_{n}^{(1)}$ :

Proposition 4.1 In the situation of Proposition 3.6, if $\Gamma$ is of type $A_{n}^{(1)}$, then $\mu_{k}(\Gamma k)$ is one of the following diagrams: $A_{n}^{(1)}, D_{n}^{(1)}, D_{n}^{(1)}(m, r), D_{n}^{(1)}(r), D_{n}^{(1)}(m, r, s)$.

\subsection{Proof of Proposition 4.1}

Let us index the vertices in $\Gamma$ by $\{1, \ldots, n\}$. Let us also write

$$
\{i \in \Gamma: k \text { is connected to } i\}=\left\{i_{1}, \ldots, i_{r}\right\}
$$

where $1 \leq i_{1}<i_{2}<\ldots<i_{r} \leq n$ and $r \geq 2$. Since $k$ is not contained in any non-oriented cycle in $\Gamma k$, the number $r$ is even.

We prove the lemma using a case by case analysis as follows:

Case 1. $r \geq 8$. In this case the subdiagram with the vertices $\left\{i_{1}, i_{1}+1, i_{4}, i_{4}+1, i_{7}, k\right\}$ is always mutation equivalent to $E_{6}$ (Fig. 4), contradicting (3.2).

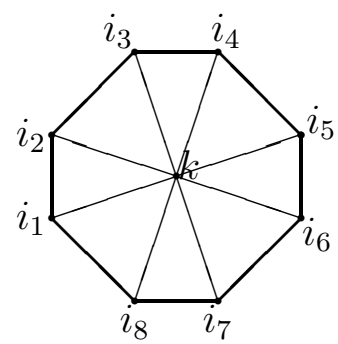

Figure 4: The subdiagram on vertices $\left\{i_{1}, i_{2}, i_{4}, i_{5}, i_{7}, k\right\}$ is mutation equivalent to $E_{6}$

Case 2. $r=6$.

Subcase 2.1. $\Gamma$ has length 6 . Then the subdiagram with the vertices $\left\{i_{1}, i_{2}, \ldots, i_{5}, k\right\}$ is mutation equivalent to $E_{6}$ (Fig. 5), contradicting (3.2).

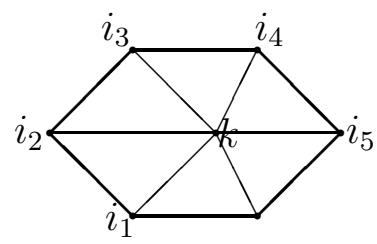

Figure 5: The subdiagram on vertices $\left\{i_{1}, i_{2}, \ldots, i_{5}, k\right\}$ is mutation equivalent to $E_{6}$

Subcase 2.2. $\quad \Gamma$ has length greater than 6 . Let us note that $k$ is contained in a cycle $C \subset \Gamma k$ of length greater than 3 . 
Subsubcase 2.2.1. $k$ is contained in a cycle $C \subset \Gamma k$ of length equal to 4. Let us assume, without loss of generality, that $C=\left[k, i_{1}=1,2, i_{2}=3\right]$ and $k$ is not connected to the vertex 2 . Then the subdiagram with the vertices $C \cup\left\{n, i_{5}\right\}$ in $\Gamma k$ is mutation equivalent to $E_{6}$ (Fig. 6), contradicting (3.2).

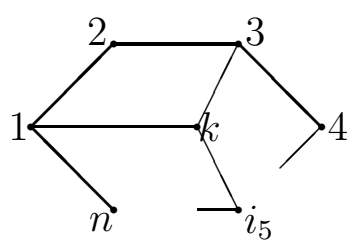

Figure 6: The subdiagram on vertices $\left\{k, 1,2,3, n, i_{5}\right\}$ is mutation equivalent to $E_{6}$

Subsubcase 2.2.2. $\quad k$ is contained in a cycle $C \subset \Gamma k$ of length greater than 4. Let us assume, without loss of generality, that $C=\left[k, i_{1}=1,2, \ldots, r, i_{2}\right]$ where $p \geq 3$ and $k$ is not connected to any vertex in $\{2, \ldots, p\}$. Then the subdiagram with the vertices $\left\{k, 1,2, p, i_{2}, i_{4}\right\}$ is mutation equivalent to $E_{6}$ (Fig. 7), contradicting (3.2).

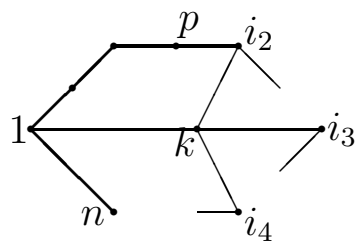

Figure 7: The diagram on vertices $\left\{k, 1,2, p, i_{2}, i_{4}\right\}, p \geq 3$, contains (a subdiagram mutation equivalent to) $E_{6}$

Case 3. $\quad r=4$. Let us first assume that the diagram $\Gamma k$ does not contain a triangle. Then, e.g., the subdiagram with the vertices $\left\{k, i_{1}, i_{1}+1, i_{2}, i_{2}+1, i_{4}\right\}$ is always mutation equivalent to $E_{6}$ (Fig. 8), contradicting (3.2). Let us now assume that $\Gamma k$ contains at least one triangle and consider the subcases.

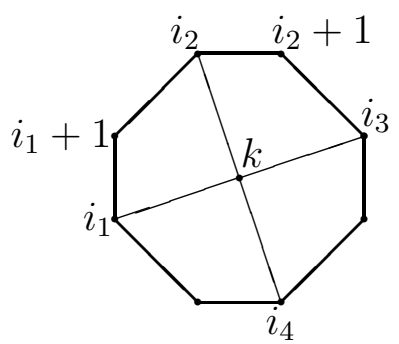

Figure 8: The subdiagram on vertices $\left\{k, i_{1}, i_{1}+1, i_{2}, i_{2}+1, i_{4}\right\}$ is mutation equivalent to $E_{6}$

Subcase 3.1. The diagram $\Gamma k$ contains precisely one triangle. 
Subsubcase 3.1.1. The vertex $k$ is contained in a cycle $C^{\prime} \subset \Gamma k$ of length greater than 4. Let us assume without loss of generality that $C^{\prime}=\left[k, i_{1}=1,2, \ldots, i_{2}=r\right]$. Since $\Gamma k$ contains precisely one triangle, we may assume that $i_{3}$ is not connected to $i_{2}$. Then the subdiagram with the vertices $C^{\prime} \cup\left\{i_{3}\right\}$ is mutation equivalent to $E_{6}$, contradicting (3.2). Subsubcase 3.1.2. The vertex $k$ is not contained in any cycle $C^{\prime} \subset \Gamma k$ such that $C^{\prime}$ has length greater than 4 . Let us first assume, without loss of generality, that $T=\left[k, i_{1}=\right.$ $\left.1, i_{2}=2\right]$ is the unique triangle in $\Gamma k$. Since $k$ is not contained in a cycle of length greater than 4 , we have $i_{3}=4$ and $i_{5}=6$. Then the subdiagram with the vertices $\{k, 1,2,3,4,6\}$ is mutation equivalent to $E_{6}$ (Fig. 9), contradicting (3.2).

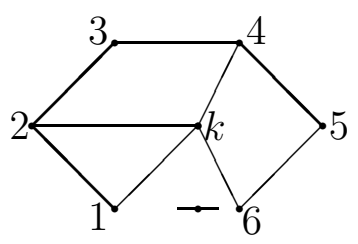

Figure 9: The subdiagram on vertices $\{k, 1,2,3,4,6\}$ is mutation equivalent to $E_{6}$

Subcase 3.2. The diagram $\Gamma k$ contains precisely two triangles, say $T_{1}$ and $T_{2}$.

Subsubcase 3.2.1. $\quad T_{1}$ and $T_{2}$ share a common edge. Let us assume, without loss of generality, that $T_{1}=\left[k, i_{1}=1, i_{2}=2\right]$ and $T_{2}=\left[k, i_{2}=2, i_{3}=3\right]$. Then $k$ is not connected to any vertex in $\{4, n\}$, thus the subdiagram with the vertices $T_{1} \cup T_{2} \cup\{k, 4, n\}$ (Fig. 10) is mutation equivalent to $E_{6}$, contradicting (3.2).

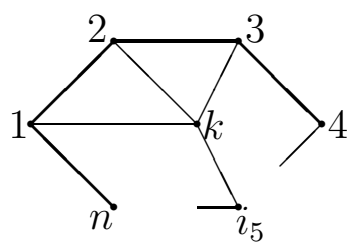

Figure 10: The subdiagram on vertices $\{n, 1,2,3,4, k\}$ is mutation equivalent to $E_{6}$

Subsubcase 3.2.2. $\quad T_{1}$ and $T_{2}$ do not share a common edge. Then $\mu_{k}(\Gamma k)$ is of type $D_{n}^{(1)}(m, r, s)$.

Subcase 3.3. The diagram $\Gamma k$ contains precisely three triangles, say $T_{1}, T_{2}$ and $T_{3}$. Then the diagram $\mu_{k}(\Gamma k)$ is of type $D_{n}^{(1)}(m, r)$.

There is no other subcases in Case 3.

Case 4. $r=2$.

Subcase 4.1. The vertices $i_{1}$ and $i_{2}$ are connected to each other. Then $\mu_{k}(\Gamma k)$ is of type $A_{n}^{(1)}$.

Subcase 4.2. The vertices $i_{1}$ and $i_{2}$ are not connected to each other. Then the vertex $k$ is contained in precisely two cycles, say $C_{1}$ and $C_{2}$ in $\Gamma k$. We may assume, without loss of generality, that the length of $C_{1}$ is less than or equal to the length of $C_{2}$. 
Subsubcase 4.2.1. The cycle $C_{1}$ has length 4. Then $\mu_{k}(\Gamma k)$ is of type $D_{n}^{(1)}(r)$.

Subsubcase 4.2.2. The cycle $C_{1}$ has length greater than 4. Let us assume, without loss of generality that, $i_{1}=1$ and $i_{2} \geq 4$. Then the subdiagram with the vertices $\left\{k, i_{2}-2, i_{2}-1, i_{2}, i_{2}+1, i_{2}+2\right\}$ is an $E_{6}$, contradicting (3.2).

\subsection{Proof of Proposition 3.6}

Let us note that for $\Gamma$ which is of type $A_{n}^{(1)}$ the proposition follows from Proposition 4.1. We need to show that the proposition holds for each of the remaining diagrams $D_{n}^{(1)}$, $D_{n}^{(1)}(m, r), D_{n}^{(1)}(r), D_{n}^{(1)}(m, r, s)$. This can be done in exactly the same way as we did for $A_{n}^{(1)}$. Not to repeat the same arguments, we do not give the proof here; we refer to the corresponding section (Section 4.2) in the long version of this paper [12] (or to [13]).

\section{Simply-laced minimal 2-infinite diagrams with at most 9 vertices}

In this section we will characterize simply-laced 2-finite diagrams using basic moves, a class of graph transformations. Using this characterization we will prove Proposition 3.7 in Section 5.5.

Definition 5.1 Suppose that $\bar{\Gamma}$ is a weightless undirected graph and that a, c are two vertices which are connected to each other. The basic move $\phi_{c, a}$ is the transformation that changes $\bar{\Gamma}$ as follows: it connects $c$ to vertices that are connected to a but not connected to $c$; at the same time it disconnects vertices from $c$ if they are connected to $a$. We call two graphs $\bar{\Gamma}$ and $\bar{\Gamma}^{\prime} \mathrm{BM}$ (basic move)-equivalent if they can be obtained from each other by a sequence of basic moves.

For a diagram $\Gamma$, we denote by $\bar{\Gamma}$ the undirected graph which is defined as follows: the vertex set of $\bar{\Gamma}$ is the same as that of $\Gamma$ and two vertices $i, j$ in $\bar{\Gamma}$ are connected to each other if and only if they are connected in $\Gamma$ by an edge whose weight is an odd integer. By a subgraph of $\bar{\Gamma}$, we mean a graph obtained from $\bar{\Gamma}$ by taking an induced subgraph on a subset of vertices.

Basic moves were introduced in [2]. Here we note that they are related to mutations as follows:

Proposition 5.2 Suppose that a diagram $\Gamma$ is mutation equivalent to $\Gamma^{\prime}$. Then $\bar{\Gamma}$ is BM-equivalent to $\bar{\Gamma}^{\prime}$.

Proof. It is enough to establish the proposition for $\Gamma^{\prime}=\mu_{k}(\Gamma)$ where $k$ is an arbitrary vertex in $\Gamma$. Let us assume that $c_{1}, c_{2}, \ldots, c_{r}$ are the vertices which are connected to $k$ by a directed edge pointing towards $k$ with an odd weight. Then $\bar{\Gamma}^{\prime}=\phi_{c_{r}, k} \circ \ldots \phi_{c_{2}, k} \circ \phi_{c_{1}, k}(\bar{\Gamma})$. 
We observe that the converse of Proposition 5.2 holds for 2-finite diagrams. More precisely:

Theorem 5.3 Suppose that $\Gamma$ is a simply-laced connected diagram that does not contain any non-oriented cycle. Let $\bar{\Gamma}$ be the underlying undirected graph of $\Gamma$. Then the diagram $\Gamma$ is 2-finite if and only if $\bar{\Gamma}$ is BM-equivalent to a Dynkin graph.

We prove the theorem in Section 5.3 after some preparation.

\subsection{Basic moves as linear transformations}

In this subsection we give some definitions related to basic moves; the details can be found in [11]. Let $\bar{\Gamma}$ be a connected graph with vertex set $I$. We denote by $V$ the vector space with the basis $I$. The graph $\bar{\Gamma}$ naturally defines an alternating $\mathbb{F}_{2}$-valued bilinear form $\Omega$ on $V$ as follows: for any $i, j \in I, \Omega(i, j)=1$ if and only if $i$ and $j$ are connected to each other in $\bar{\Gamma}$. Then a basic move $\phi_{c, a}$ corresponds to the following change of basis for $\Omega$ :

$$
\phi_{c, a}(I)=I-\{c\} \cup\{c+a\} .
$$

For any vector subspace $U$ of $V$, we denote by $U_{0}$ the kernel of the restriction of $\Omega$ on $U$, i.e. $U_{0}=\left\{u \in U: \Omega\left(u, u^{\prime}\right)=0\right.$ for all $\left.u^{\prime} \in U\right\}$. We denote by $Q$ the $\mathbb{F}_{2}$-valued quadratic form which is defined as follows: $Q(u+v)=Q(u)+Q(v)+\Omega(u, v),(u, v \in V)$ and $Q(i)=1$ for all $i \in I$.We define $U_{00}=U_{0} \cap Q^{-1}(0)$. Clearly $U_{00}$ is a vector subspace of $U_{0}$. If $V_{0}=V_{00}$, then the Arf invariant of $Q$ is defined as follows: $\operatorname{Arf}(Q)=\sum Q\left(e_{i}\right) Q\left(f_{i}\right)$ where $\left\{e_{1}, f_{1}, \ldots, e_{r}, f_{r}, h_{1}, \ldots, h_{p}\right\}$ is a symplectic basis, i.e. a basis such that $\Omega\left(e_{i}, f_{i}\right)=1$ and the rest of the values of $\Omega$ are 0 .

We will use the following simple fact in our proof of Theorem 5.3:

Proposition 5.4 Suppose that $U$ is a vector subspace of codimension one in $V$. Then $\operatorname{dim}\left(V_{00}\right) \geq \operatorname{dim}\left(U_{00}\right)-1$.

Let $v \in V$ be a non-zero vector which is not in $U$. Let us assume that $K=\left\{x_{1}, \ldots, x_{p}\right\}$ is a basis of $U_{00}$ and write $r(K)=\#\left\{x_{i} \in K: \Omega\left(v, x_{i}\right)=1\right\}$. If $r(K)=0$, then $K$ is also a basis for $V_{00}$, thus $\operatorname{dim}\left(V_{00}\right)=\operatorname{dim}\left(U_{00}\right)$. Suppose that $r(K)=1$ and $\Omega\left(v, x_{i}\right)=1$. Then $K-\left\{x_{i}\right\}$ is a basis for $V_{00}$. Let us now assume that $r(K)>1$ and, assume without loss of generality, that $\Omega\left(v, x_{i}\right)=1$ for $i=1,2$. Then $K_{1}=\left\{x_{1}, x_{1}+x_{2}, x_{3}, \ldots, x_{p}\right\}$ is also a basis for $U_{00}$ while $r\left(K_{1}\right)=r(K)-1$ (because $\Omega\left(v, x_{1}+x_{2}\right)=0$ ), thus the proposition follows by induction.

\subsection{Basic moves and Dynkin graphs}

In this subsection we give some properties of graphs which are BM-equivalent to Dynkin graphs and discuss their implications on mutations. Throughout this subsection, we assume that $\bar{\Gamma}$ is a connected graph in the set-up of (sub)section 5.1. 
Proposition 5.5 Suppose that $\bar{\Gamma}$ contains $E_{6}$. If the number of vertices in $\bar{\Gamma}$ is greater than or equal to 9 , then it is not BM-equivalent to any Dynkin graph.

Since $\bar{\Gamma}$ contains $E_{6}$, any tree which is BM-equivalent to $\bar{\Gamma}$ also contains $E_{6}$ [11, Theorem 2.7]. Since no Dynkin graph with 9 or more vertices contains $E_{6}$, the graph $\bar{\Gamma}$ can not be equivalent to any Dynkin graph.

Corollary 5.6 Suppose that $\Gamma$ is a simply-laced diagram that contains a subdiagram which is mutation equivalent to $E_{6}$. If the number of vertices in $\Gamma$ is greater than or equal to 9 , then $\Gamma$ is 2-infinite.

Proposition 5.7 In the set-up of Section 5.1, we have the following description of the BM-equivalence classes of the simply-laced Dynkin graphs with 6, 7, or 8 vertices:

- Suppose that $\bar{\Gamma}$ has precisely 6 vertices. Then $\bar{\Gamma}$ is BM-equivalent to a Dynkin graph if and only if one of the following holds:

(a) $V_{0}=\{0\}$,

(b) $\operatorname{dim}\left(V_{0}\right)=2$ and $V_{0} \neq V_{00}$.

- Suppose that $\bar{\Gamma}$ has precisely 7 vertices. Then $\bar{\Gamma}$ is BM-equivalent to a Dynkin graph if and only if $\operatorname{dim}\left(V_{0}\right)=1$ and one of the following holds:

(a) $V_{0} \neq V_{00}$,

(b) $V_{0}=V_{00}$ and $\operatorname{Arf}(Q)=0$

- Suppose that $\bar{\Gamma}$ has precisely 8 vertices. Then $\bar{\Gamma}$ is BM-equivalent to a Dynkin graph if and only if one of the following holds:

(a) $V_{0}=\{0\}$ and $\operatorname{Arf}(Q)=0$.

(b) $\operatorname{dim}\left(V_{0}\right)=1$ and $\bar{\Gamma}$ is BM-equivalent to the Dynkin graph $D_{8}$. (Note that an explicit description of graphs which are BM-equivalent to $D_{8}$ is given in [11, Theorems 2.7, 2.10]).

This is a special case of [2, Theorem 4.1] and [9, Theorem 3.8] (see also [11, Theorem 2.7]).

Proposition 5.8 Suppose that $\bar{\Gamma}$ contains a subgraph which is BM-equivalent to $E_{6}$. If $\operatorname{dim}\left(V_{00}\right) \geq 1$, then $\bar{\Gamma}$ is not BM-equivalent to any Dynkin graph.

This statement is also a special case of [9, Theorem 3.8] and [11, Theorem 2.7].

Proposition 5.9 Suppose that $\bar{\Gamma}$ contains a subgraph $X$ which is $E_{6}^{(1)}$ or $E_{7}^{(1)}$. Then $\bar{\Gamma}$ is not BM-equivalent to any Dynkin graph. 
If the number of vertices in $\bar{\Gamma}$ is greater than or equal to 9 , then the statement follows from Proposition 5.5 because $X$ contains $E_{6}$ as a subgraph. Let us now assume that $\Gamma$ has at most 8 vertices. If $X$ is $E_{7}^{(1)}$, then $X=\bar{\Gamma}$ which is not BM-equivalent to any Dynkin graph by [9]. Let us now assume that $X$ is $E_{6}^{(1)}$ indexed as in Fig 11 . Then $\bar{\Gamma}$ can not be BM-equivalent to any Dynkin graph with seven vertices [11, Theorem 2.3], so we may assume that $\bar{\Gamma}$ has precisely 8 vertices. We may also assume that $V_{0}=0$, otherwise $\bar{\Gamma}$ is not equivalent to any Dynkin graph by Proposition 5.8. Under these assumptions, by Proposition 5.7, the graph $\bar{\Gamma}$ is BM-equivalent to a Dynkin graph if and only if $\operatorname{Arf}(Q)=0$. We will establish a symplectic basis and show that $\operatorname{Arf}(Q)=1$, which will prove the proposition. We note that the set

$$
\left\{e_{1}=a_{1}, f_{1}=a_{2}, e_{2}=a_{4}, f_{2}=a_{5}, e_{3}=a_{6}, f_{3}=a_{7}, e_{4}=a_{1}+a_{3}+a_{5}+a_{7}\right\}
$$

could be completed to a symplectic basis, i.e. there is a non-zero vector $f_{4} \in V$ such that $\Omega\left(e_{4}, f_{4}\right)=1$ and $\Omega\left(e_{i}, f_{4}\right)=\Omega\left(f_{i}, f_{4}\right)=0$ for $i=1,2,3$, which gives $\operatorname{Ar} f(Q)=1$ because $Q\left(a_{1}+a_{3}+a_{5}+a_{7}\right)=0$ and $Q\left(a_{i}\right)=1$ for $i=1, \ldots, 7$.

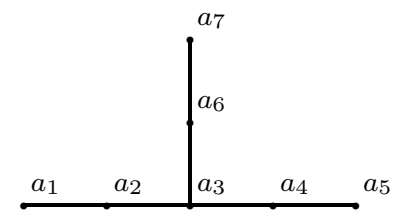

Figure 11: The extended Dynkin graph $E_{6}^{(1)}$

Proposition 5.10 If $\bar{\Gamma}$ contains a subgraph $X$ which is of type $D_{n}^{(1)}$, then it is not BMequivalent to any Dynkin graph.

Let us denote by $U \subset V$ the vector subspace which is spanned by the vertices in $X$ (recall that we work in the set-up of Section 5.1). By [11, Proposition 3.1], we have the following: if $X$ is a $D_{4}^{(1)}$ (resp. $\left.D_{n}^{(1)}, n \geq 5\right)$, then $\operatorname{dim}\left(U_{00}\right)=3\left(\operatorname{resp} \cdot \operatorname{dim}\left(U_{00}\right) \geq 2\right)$. If $\bar{\Gamma}$ does not contain any subgraph which is BM-equivalent to $E_{6}$, then $\operatorname{dim}\left(V_{00}\right) \geq 2$ by $[11$, Theorem 2.9], implying the conclusion of the proposition by [11, Theorem 2.3,2.7]. Let us now assume that $\bar{\Gamma}$ contains a subgraph which is BM-equivalent to $E_{6}$. If $\bar{\Gamma}$ has at least 9 vertices, then the statement follows from Proposition 5.5. If $\bar{\Gamma}$ has 6 or 7 vertices, then $\operatorname{dim}\left(U_{00}\right) \geq 1$ by Proposition 5.4, so the proposition follows from Proposition 5.8. If $\bar{\Gamma}$ has 8 vertices, then it contains a subgraph which is BM-equivalent to $E_{6}^{(1)}$ by the classification of graphs under basic moves [11, Theorems 2.3, 2.7] and [9, Theorem 3.8], thus the proposition follows from Proposition 5.9.

Finally, we will need the following statement on mutations.

Proposition 5.11 Let $C$ be a diagram which is a non-oriented cycle whose length is less than or equal to 7 and let $C k$ be a diagram obtained by adjoining a vertex $k$ to $C$. 
Suppose that $k$ is a vertex connected to at least two vertices of $C$. Suppose also that $k$ is not contained in any non-oriented cycle in $C k$, in particular $k$ is connected to an even number of vertices in $C$. Then $C k$ is mutation equivalent to a simply-laced extended Dynkin diagram.

If $C k$ does not contain any subgraph which is mutation equivalent to $E_{6}$, then the statement is the same as Proposition 4.1. If $C k$ contains a subgraph which is mutation equivalent to $E_{6}$, then $C k$ is one of the diagrams in Fig.12. It follows from a direct check that $C k$ is mutation equivalent to a simply-laced extended Dynkin diagram. (In fact, the diagram $\mu_{k}(C k)$ is in Table 4 or 5$)$. We will prove in Lemma 6.1 that each diagram in Table 4 (resp. Table 5 ) is mutation equivalent to $E_{6}^{(1)}$ (resp. $E_{7}^{(1)}$ ).

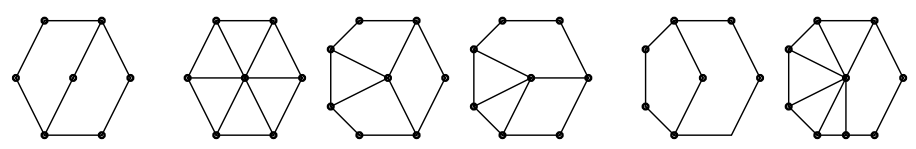

Figure 12: The diagram $C k$ of Proposition $5.11 ; k$ is the vertex in the center

\subsection{Proof of Theorem 5.3}

In view of Proposition 5.2, it is enough to prove that

$\left(^{*}\right)$ if $\Gamma$ is not 2-finite, then $\bar{\Gamma}$ is not BM-equivalent to any Dynkin graph.

To establish $(*)$, let us first notice that there is a sequence of mutations $\mu_{1}, \ldots, \mu_{k}, k \geq 1$ and a simply-laced diagram $\Gamma^{\prime}$ such that

(i) $\Gamma^{\prime}=\mu_{k} \circ \ldots \circ \mu_{1}(\Gamma)$,

(ii) the diagram $\Gamma^{\prime}$ contains a non-oriented cycle $C$,

(iii) for any $i: 1, \ldots, k-1$, the diagram $\Gamma_{i}=\mu_{i} \circ \ldots \circ \mu_{1}(\Gamma)$ is simply-laced and it does not contain any non-oriented cycle.

Let us note that $k$ is not contained in $C$ and it is connected to an even number of vertices in $C$ (because $\mu_{k}\left(\Gamma^{\prime}\right)=\Gamma_{k-1}$ is a simply-laced diagram which does not contain any nonoriented cycle). We denote by $C k$ the subdiagram with the vertices $C \cup k$. Let us first assume that $C$ has length less than or equal to 7 . Then $\mu_{k}(C k) \subset \Gamma_{k-1}$ is mutation equivalent to an extended Dynkin diagram, say $X$, (Proposition 5.11). Note that $\overline{C k}$ is BM-equivalent to $\bar{X}$ by Proposition 5.2. Since $\Gamma_{k-1}$ does not contain any non-oriented cycle, the diagram $X$ is one of the following: $D_{n}^{(1)}, E_{6}^{(1)}, E_{7}^{(1)}$. Thus $\left(^{*}\right)$ follows from Proposition 5.10 and Proposition 5.9. Let us now assume that the length of $C$ is greater than or equal to 8 . If $C k$ does not contain any subdiagram which is mutation equivalent to $E_{6}$, then $C k$ is mutation equivalent to $D_{n}^{(1)}$ by Proposition 3.6, so $\left(^{*}\right)$ follows from Proposition 5.10; otherwise it follows from Proposition 5.5. 
Corollary 5.12 Suppose that $\Gamma$ is a diagram that contains (a subdiagram which is mutation equivalent to) $E_{6}$. Let $\Gamma^{\prime}$ be a simply-laced diagram which is mutation equivalent to $\Gamma$. Suppose also that $\Gamma^{\prime}$ does not contain any non-oriented cycle. Then $\Gamma$ contains a subdiagram which is mutation equivalent to $E_{6}$.

By [11, Theorem 2.7], the diagram $\bar{\Gamma}^{\prime}$ contains a subgraph, say $\bar{E}$, which is BM-equivalent to $E_{6}$. Since $\overline{\Gamma^{\prime}}$ does not contain any non-oriented cycle, the subdiagram $E$ is mutation equivalent to (an orientation of) $E_{6}$ by the theorem.

Corollary 5.13 Suppose that $\Gamma$ is a simply-laced 2-finite diagram. Suppose also that $\Gamma$ contains a subdiagram which is mutation equivalent to $E_{6}$. Then $\Gamma$ is mutation equivalent to one of the following diagrams: $E_{6}, E_{7}, E_{8}$.

The statement immediately follows from Corollary 5.12 (and the classification of 2-finite diagrams).

Corollary 5.14 Suppose that $\Gamma$ is a simply-laced minimal 2-infinite diagram that does not contain any subdiagram which is mutation equivalent to $E_{6}$. Then $\Gamma$ is in Table 1 , i.e. $\Gamma$ is one of the following diagrams: $A_{n}^{(1)}, D_{n}^{(1)}, D_{n}^{(1)}(m, r), D_{n}^{(1)}(r), D_{n}^{(1)}(m, r, s)$.

If $\Gamma$ contains a non-oriented cycle $C$, then $\Gamma=C$ (i.e. of type $A_{n}^{(1)}$ ) because $\Gamma$ is minimal 2 -infinite. Let us now assume that $\Gamma$ does not contain any non-oriented cycle. Then there is a sequence of mutations $\mu_{1}, \ldots, \mu_{k}, k \geq 1$ and a simply-laced diagram $\Gamma^{\prime}$ such that

(i) $\Gamma^{\prime}=\mu_{k} \circ \ldots \circ \mu_{1}(\Gamma)$,

(ii) the diagram $\Gamma^{\prime}$ contains a non-oriented cycle $C$,

(iii) for any $i: 1, \ldots, k-1$, the diagram $\Gamma_{i}=\mu_{i} \circ \ldots \circ \mu_{1}(\Gamma)$ is simply-laced and it does not contain any non-oriented cycle.

We note that $k$ is not contained in $C$ and it is connected to an even number of vertices in $C$ (because $\mu_{k}\left(\Gamma^{\prime}\right)=\Gamma_{k-1}$ is a simply-laced diagram which does not contain any nonoriented cycle). We also note that, for any $i: 1, \ldots, k-1$, the diagram $\Gamma_{i}$ does not contain any subdiagram which is mutation equivalent to $E_{6}$ (Corollary 5.12). This implies that the vertex $k$ is not contained in any subdiagram which is mutation equal to $E_{6}$ in $C k$ (otherwise $\mu_{k}(C k) \subset \mu_{k}\left(\Gamma^{\prime}\right)=\Gamma_{k-1}$ contains one). By Proposition 4.1, the diagram $\mu_{k}(C k)$ is one of the diagrams $D_{n}^{(1)}, D_{n}^{(1)}(m, r), D_{n}^{(1)}(r), D_{n}^{(1)}(m, r, s)$. By Proposition 3.6 and Lemma 3.4, for any $j=1, \ldots, k-1$, the diagram $\Gamma^{j}=\mu_{k-j} \circ \ldots \mu_{k-1} \circ \mu_{k}\left(\Gamma^{\prime}\right)$, in particular $\Gamma^{k-1}=\Gamma$, contains one of the diagrams $D_{n}^{(1)}, D_{n}^{(1)}(m, r), D_{n}^{(1)}(r), D_{n}^{(1)}(m, r, s)$. Since $\Gamma$ is minimal 2-infinite, the diagram $\Gamma$ must be one of $D_{n}^{(1)}, D_{n}^{(1)}(m, r), D_{n}^{(1)}(r)$, $D_{n}^{(1)}(m, r, s)$. 
Corollary 5.15 A simply-laced diagram $\Gamma$ is mutation equivalent to the Dynkin diagram of type $A$ if and only if it does not have subdiagrams of the following form:

a non-oriented cycle,

a tree of type $D_{4}$,

two triangles sharing a common edge,

any (oriented) cycle whose length is greater than or equal to 4.

In view of Theorem 5.3, the corollary follows from [11, Theorem 2.7].

Corollary 5.16 Suppose that $\Gamma$ is a simply-laced minimal 2-infinite diagram. Suppose also that $\Gamma$ contains a subdiagram which is mutation equivalent to $E_{6}$. Then $\Gamma$ is one of the diagrams in Tables 4-6 in Section 8.

We obtained this corollary by implementing the following algorithm.

\subsection{Algorithm to compute minimal 2-infinite diagrams that con- tains a subdiagram which is mutation equivalent to $E_{6}$}

Let us denote the set of those diagrams by $\mathbf{E}$. Let $\Gamma \in \mathbf{E}$ be a diagram with $n$ vertices. Then $7 \leq n \leq 9$ by Corollary 5.6. If $n=8$ or $n=9$, then $\Gamma$ contains a subdiagram which is mutation equivalent to $E_{n-1}^{(1)}$ (Corollary 5.13). Thus we can compute $\mathbf{E}$ as follows:

For $n=7,8,9$ :

- compute the mutation class of $E_{n-1}$,

- for each diagram $\Gamma$ in the mutation class of $E_{n-1}$, and each non-empty subset $X$ of vertices in $\Gamma$,

- form the graph $\Gamma k$ by adjoining a vertex $k$ to $\Gamma$ as follows: $k$ is connected to all of the vertices in $X$ but not connected any other vertex in $\Gamma$,

- check if $\Gamma k$ is minimal 2-infinite using Theorem 5.3 and Proposition 5.7,

- (if $\Gamma k$ is minimal 2-infinite, check whether it is in the mutation class of $E_{n-1}^{(1)}$ ).

We implemented this algorithm using the computer algebra package Maple (v.8). Our code is available at [15].

\subsection{Proof of Proposition 3.7}

If $\Gamma$ contains a subdiagram which is mutation equivalent to $E_{6}$, then the theorem follows from Corollary 5.16. If $\Gamma$ does not contain any subdiagram which is mutation equivalent to $E_{6}$, then the theorem follows from Corollary 5.14. 


\section{$6 \quad$ Proof of Theorem 3.1}

We prove the theorem following the outline given in Section 3, i.e. by proving Lemmas 3.3, 3.4, 3.5. Since Lemma 3.5 is somewhat more involved, we will prove it in a separate section, Section 7 , for convenience.

\subsection{Proof of Lemma 3.3}

We first prove that $\Gamma$ is 2 -infinite. According to [5, Propositions 9.3, 9.7]), any nonoriented oriented cycle is 2-infinite. In particular any diagram in Table 2 is 2 -infinite. By [5, Propositions 9.3, 9.7]), any extended Dynkin diagram is also 2-infinite. Thus for $\Gamma$ which is not in Table 2 the fact that it is 2-infinite follows from the following stronger statement:

Lemma 6.1 Suppose that $\Gamma$ in Section 8 is not one of the diagrams in Table 2. Then $\Gamma$ is mutation equivalent to an extended Dynkin diagram (Fig. 3).

We prove the lemma using a case by case analysis. Throughout the proof, we assume that $\Gamma$ is indexed as in the associated table (Section 8).

Case 1. $\Gamma$ is in Table 1.

Subcase 1.1. $\Gamma$ is of type $B_{n}^{(1)}(m, r)$ (resp. $B_{n}^{(1)}(r)$ ). Then we have the following: if $r=3$, then $\mu_{b_{1}}(\Gamma)$ is of type $B_{n}^{(1)}$; if $r>3$, then $\mu_{b_{1}}(\Gamma)$ is of type $B_{n}^{(1)}(m+1, r-1)$ (resp. $\left.B^{(1)}(1, r-1)\right)$, so it is mutation equivalent to $B_{n}^{(1)}$ by induction on $r$.

Subcase 1.2. $\Gamma$ is of type $D_{n}^{(1)}(m, r)$. Then we have the following: if $r=3$, then $\mu_{c_{1}}(\Gamma)$ is of type $D_{n}^{(1)}$; if $r>3$, then $\mu_{c_{1}}(\Gamma)$ is of type $D_{n}^{(1)}(m+1, r-1)$, so it is mutation equivalent to $D_{n}^{(1)}$ by induction on $r$.

Subcase 1.3. $\Gamma$ is of type $D_{n}^{(1)}(r)$. Then we have the following: if $r=3$, then $\mu_{b_{1}}(\Gamma)$ is of type $D_{4}^{(1)}$; if $r>3$, then $\mu_{b_{1}}(\Gamma)$ is of type $D_{n}^{(1)}(1, r-1)$ which is mutation equivalent to $D_{n}^{(1)}$ by Subcase 1.1 .

Subcase 1.4. $\Gamma$ is of type $D_{n}^{(1)}(m, r, s)$. Then we have the following: if $r=3$, then $\mu_{c_{1}}(\Gamma)$ is of type $D_{n}^{(1)}(m+1, s)$, which is mutation equivalent to $D_{n}^{(1)}$ by Subcase 1.2; if $r>3$, then $\mu_{c_{1}}(\Gamma)$ is of type $D_{n}^{(1)}(m+1, r-1, s)$, so it is mutation equivalent to $D_{n}^{(1)}$ by induction on $r$.

Case 2. $\Gamma$ is in Table 3.

Subcase 2.1. $\Gamma$ contains a triangle, say $T$, which is not adjacent to any cycle. Then $T$ contains a vertex, say $k$, which is adjacent to precisely two edges one of them being weightless. Then the mutation $\mu_{k}$ eliminates the triangle $T$. Continuing this process one has $F_{4}^{(1)}$ or $G_{2}^{(1)}$.

Subcase 2.2. Subcase 2.1 does not hold. In this case $\Gamma$ has two cycles and there is a vertex $k$ which is contained in both cycles. If the cycles are triangles, then $\mu_{k}(\Gamma)$ is as in Subcase 2.1; otherwise $\mu_{k}(\Gamma)$ has two adjacent triangles, and applying this case once more will reduce it to the Subcase 2.1. 
Case 3. $\Gamma$ is in Tables 4-6. Then, it follows from a computer check that $\Gamma$ is mutation equivalent to $E_{6}^{(1)}, E_{7}^{(1)}$ or $E_{8}^{(1)}$ [15].

We are done with the proof of Lemma 6.1, thus have shown that any diagram $\Gamma$ in Section 8 is 2-infinite. Now we will prove that $\Gamma$ is minimal:

Lemma 6.2 Suppose that $\Gamma$ is an arbitrary diagram in Section 8. Then any connected subdiagram $\Gamma^{\prime}$ obtained from $\Gamma$ by deleting a vertex $b$ is mutation equivalent to a Dynkin diagram.

We prove the lemma using a case by case analysis. Throughout the proof, we assume that $\Gamma$ is indexed as in the associated table (Section 8).

Case 1. $\Gamma$ is in Table 1. The lemma obviously holds for the extended Dynkin diagrams and the diagrams $I_{2}(a), a \geq 4$.

Subcase 1.1. $\Gamma$ is of type $D_{n}^{(1)}(m, r)$.

Subsubcase 1.1.1. $b=b_{1}$ or $b=b_{2}$. Then the diagram $\mu_{c_{r-1} \ldots} \ldots \mu_{c_{1}}\left(\Gamma^{\prime}\right)$ is of type $D$.

Subsubcase 1.1.2. $b=c_{i}$ for some $i: 1<i<r$. Then the diagram $\mu_{c_{i-1} \ldots \mu_{c_{1}}}\left(\Gamma^{\prime}\right)$ is of type $D$.

The remaining (sub)subcases are trivial.

Subcase 1.2. $\Gamma$ is of type $D_{n}^{(1)}(r)$.

Subsubcase 1.2.1. $b=a_{1}$ or $b=c_{1}$ Let us assume, without loss of generality, that $b=a_{1}$. Then $\mu_{c_{1}}\left(\Gamma^{\prime}\right)$ is an (oriented) cycle which is mutation equivalent to a Dynkin graph of type $D[5]$.

Subsubcase 1.2.2. $b=b_{i}$ for some $i: 1<i<r$. Then the diagram $\mu_{c_{i-1}} \ldots \mu_{c_{1}}\left(\Gamma^{\prime}\right)$ is of type $D$.

The remaining (sub)subcases are trivial.

Subcase 1.3. $\Gamma$ is of type $D_{n}^{(1)}(m, r, s)$. By the same arguments as in Subcase 1.1, the diagram $\Gamma^{\prime}$ is mutation equivalent to a tree of type $A$ or $D$.

Subcase 1.4. $\Gamma$ is of type $B_{n}^{(1)}(m, r)$ or $B_{n}^{(1)}(r)$. In this case $\Gamma^{\prime}$ is mutation equivalent to a tree of type $A, B$ or $D$.

Case 2. $\Gamma$ is in Tables 2-3. In this case the lemma is almost obvious.

Case 3. $\Gamma$ is in Tables $4-6$. For this case we obtained the lemma by a computer check (see Section 5.4 and [15]).

\subsection{Proof of Lemma 3.4}

Let us also show this lemma by considering possible cases:

Case 1. $\Gamma$ is in Table 1. In this case we have one of the following: $\mu_{k}(\Gamma k)$ is in Table 1 or the subdiagram $\Gamma^{\prime} \subset \mu_{k}(\Gamma k)$ formed by all of the vertices which are different from $k$ is in Table 1. For most $k$ we observed those two statements in our proof of Lemma 6.1. For the remaining vertices they follow from a direct check. 
Case 2. $\Gamma$ is in Tables 2,3. In this case we have one of the following: $\mu_{k}(\Gamma k)$ is in Table 2,3 or the subdiagram $\Gamma^{\prime} \subset \mu_{k}(\Gamma k)$ formed by all of the vertices which are different from $k$ is in Section 8. Those two statements also follow from a direct check.

Case 3. $\Gamma$ is in Tables 4-6. In this case the lemma follows from Proposition 3.7 (because $\Gamma k$ is 2-infinite so is $\left.\left.\mu_{k}(\Gamma k)\right)\right)$.

\section{$7 \quad$ Proof of Lemma 3.5}

We have divided the proof into several subsections. In each subsection, we assume that the diagrams $\Gamma$ and $\Gamma k$ are as in the title (of the subsection). We will also assume, without loss of generality, that $k$ is connected to at least two vertices in $\Gamma$, (otherwise $\Gamma \subset \mu_{k}(\Gamma k)$, so we may take $\Gamma^{\prime}=\Gamma$ ). We will show that one of the following two statements is satisfied:

$k$ is contained in a diagram $\Gamma^{\prime \prime} \subset \Gamma k$ such that $\Gamma^{\prime \prime}$ is in Section 8, the diagram $\mu_{k}(\Gamma k)$ contains a subdiagram $\Gamma^{\prime}$ which is in Section 8

Let us note that (7.1) implies (7.2) by Lemma 3.4, so Lemma 3.5 holds in any case.

\subsection{The diagram $\Gamma k$ is simply laced}

If $\Gamma$ has at most 8 vertices, then obviously $\mu_{k}(\Gamma k)$ has at most 9 vertices and it is 2 -infinite, so we have (7.2) by Proposition 3.7. Let us now assume that $\Gamma$ has at least 9 vertices and that

$\Gamma k$ contains in a subdiagram $E \subset \Gamma k$ which is mutation

equivalent to the Dynkin diagram $E_{6}$.

We claim that there exists a connected subdiagram $X k \subset \Gamma k$ of 9 vertices which contains both $E$ and $k$. To prove this claim we argue as follows: if $\Gamma$ is in Table 1, then it follows by inspection that $\Gamma$ does not contain any subdiagram which is mutation equivalent to $E_{6}$, so the subdiagram $E$ must contain the vertex $k$ and we may take $X k$ to be any connected subdiagram which has 9 vertices and contains $E$. If $\Gamma$ is not in Table 1 , then it must belong to Table 6 with precisely 9 vertices. We note, by inspection, that $\Gamma$ contains a subdiagram which is mutation equivalent to $E_{6}$, thus in our set-up of (7.3) we may assume that $E \subset \Gamma$. Let $X \subset \Gamma$ be any (connected) subdiagram with precisely 8 vertices such that $E \subset X$. Note that $X$ is 2-finite because it is a proper subdiagram of $\Gamma$, which is minimal 2-infinite (Lemma 3.3). Since $k$ is connected to at least two vertices in $\Gamma$, it is connected to at least one vertex in $\Gamma$, so we may take $X k$ to be the subdiagram formed by $X$ and $k$. Then $X k$ is 2 -infinite by Corollary 5.6, thus it contains a subdiagram $X^{\prime}$ from Section 8 by Proposition 3.7. Since $X$ is 2 -finite, the vertex $k$ is necessarily contained in $X^{\prime}$, thus (7.1) is satisfied.

If (7.3) is not satisfied, then Lemma 3.5 follows from Proposition 3.6. 


\subsection{The diagram $\Gamma k$ is non-simply-laced while $\Gamma$ is simply-laced}

A glance at Table 2,3 shows that

if $\Gamma k$ contains an edge $e$ whose weight is greater than or equal to 3 , then $e$ is contained in a subdiagram $\Gamma^{\prime} \subset \Gamma k$ which is one of the diagrams in Table 2 or Table 3,

therefore (7.1) is satisfied. Let us now assume that the condition of (7.4) is not satisfied. Then, by Definition 2.3, any edge which contains $k$ has weight equal to 2 .

Case 1. $k$ is connected to two vertices, say $i, j$, which are not connected to each other. Then the subdiagram with the vertices $\{k, i, j\}$ is of type $C_{n}^{(1)}$.

Case 2. Case 1 does not hold. Then $k$ is connected to precisely two vertices, say $i, j$, in $\Gamma$. Furthermore, the vertices $i$ and $j$ are connected to each other.

Subcase 2.1. $\Gamma$ is of type $A_{n}^{(1)}$, i.e. a non-oriented cycle. Then the diagram $\mu_{k}(\Gamma k)$ is of type $B_{n}^{(1)}(r)$.

Subcase 2.2. $\Gamma$ is not of type $A_{n}^{(1)}$.

Subsubcase 2.2.1. The edge $[i, j]$ is contained in a cycle, say $C$, in $\Gamma$. Then the subdiagram with $C \cup\{k\}$ is of type $B_{n}^{(1)}(r)$.

Subsubcase 2.2.2. The edge $[i, j]$ is not contained in any cycle in $\Gamma$. We first note, by inspection, that $\Gamma$ contains a subdiagram $X$ which is one of the following: a tree of type $D_{4}$, two triangles sharing a common edge, a (oriented) cycle whose length is greater than or equal to 4 . Let $P$ be the shortest path that connects $k$ to $X$. Then the subdiagram with $P$ and $X$ contains a subdiagram which is of type $B_{n}^{(1)}$ or $B_{n}^{(1)}(m, r)$.

\subsection{The diagram $\Gamma$ is a non-simply-laced diagram}

We need to show Lemma 3.5 for $\Gamma$ which is a non-simply laced diagram from Tables 1-3. The proof follows by using the same arguments as in Section 7.2. To illustrate we will prove the lemma for $\Gamma$ which is of type $C_{n}^{(1)}$; for other types we refer to the corresponding sections (Sections 7.3-7.5) in the long version of this paper [12] (or refer to [13]).

To prove Lemma 3.5 for $\Gamma$ which is of type $C_{n}^{(1)}$; in view of (7.4), we assume that the maximum edge-weight in $\Gamma k$ is 2 .

Case 1. $k$ is not connected to any vertex in the set $\left\{b_{1}, c_{1}\right\}$.

Subcase 1.1. $\quad k$ is not contained in any edge whose weight is equal to 2 in $\Gamma k$. Then we have the following:

(i) Suppose that $k$ is connected to two distinct vertices $a_{i}, a_{j}$ in $\left\{a_{1}, \ldots, a_{m}\right\}$ and $a_{i}, a_{j}$ are not connected to each other by an edge. Let $r=\min \left\{a_{i}: k\right.$ is connected to $\left.a_{i}\right\}$ and $s=\max \left\{i: k\right.$ is connected to $\left.a_{i}\right\}$. Then the subdiagram with the vertices

$$
\left\{b_{1}, a_{1}, \ldots, a_{r}, k, a_{s}, \ldots, a_{m}, c_{1}\right\}
$$

is of type $C_{n}^{(1)}$ (Fig. 13). 
(ii) If (i) does not hold, then $k$ is connected to precisely two vertices $a_{i}, a_{j}$ in $\left\{a_{1}, \ldots, a_{m}\right\}$ such that $a_{i}, a_{j}$ are connected to each other by an edge. Then $\mu_{k}(\Gamma k)$ is of type $C_{n}^{(1)}$.

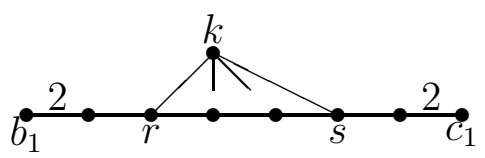

Figure 13: figure for Subcase 1.1

Subcase 1.2. $\quad k$ is contained in an edge whose weight is equal to 2. Then for any $a_{i}$ connected to $k$, the edge $\left[k, a_{i}\right]$ is weighted 2 . Let $P$ be the shortest path that connects $k$ to $c_{1}$. Then the subdiagram with the vertices $P$ is of type $C_{n}^{(1)}$ (Fig. 14).

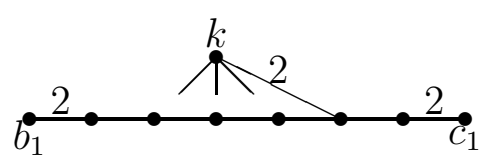

Figure 14: figure for Subcase 1.2

Case 2. $\quad k$ is connected to precisely one vertex, say $b_{1}$, in the set $\left\{b_{1}, c_{1}\right\}$. We note that the vertex $k$ is connected to at least one vertex in $\left\{a_{1}, \ldots, a_{m}\right\}$.

Subcase 2.1. The edge $\left[k, b_{1}\right]$ is weightless. We note that for any vertex $a$ in $\left\{a_{1}, \ldots, a_{m}\right\}$ such that $k$ is connected to $a$, the edge $[a, k]$ is weighted 2. Let us write $s=\max \{i$ : $k$ is connected to $\left.a_{i}\right\}$. Then the diagram $\Gamma^{\prime}$ on vertices $\left\{k, a_{s}, \ldots, a_{m}, c_{1}\right\}$ is of type $C_{n}^{(1)}$.

Subcase 2.2. The edge $\left[k, b_{1}\right]$ is weighted. We note that, for any vertex $a$ in $\left\{a_{1}, \ldots, a_{m}\right\}$ such that $k$ is connected to $a$, the edge $[a, k]$ is weightless. Then we have the following:

- If $k$ is not connected to $a_{1}$, then the subdiagram with $\left\{k, b_{1}, a_{1}\right\}$ is of type $C_{n}^{(1)}$

- Suppose that $k$ is connected to $a_{1}$. If $k$ is not connected to any vertex in $A=$ $\left\{a_{2}, \ldots, a_{m}\right\}$, then $\mu_{k}(\Gamma k)$ is a $C_{n}^{(1)}$. Let us now assume that $k$ is connected to a vertex in $A$ and let $s=\min \left\{i: k\right.$ is connected to $\left.a_{i} \in A\right\}$. Then the diagram on vertices $\left\{k, b_{1}, a_{1}, \ldots, a_{s}\right\}$ is a $B_{n}^{(1)}(r)$.

Case 3. $k$ is connected to $b_{1}$ and $c_{1}$. Let us first assume that the edge $\left[k, b_{1}\right]$ has weight equal to 2 . Then the edge $\left[k, c_{1}\right]$ has also weight equal to 2 , so the subdiagram with the vertices $\left\{b_{1}, k, c_{1}\right\}$ is of type $C_{n}^{(1)}$. Let us now assume that the edge $\left[k, b_{1}\right]$, hence $\left[k, c_{1}\right]$, is weightless and consider subcases.

Subcase 3.1. $\quad k$ is connected to a vertex in $A=\left\{a_{1}, \ldots, a_{m}\right\}$. We note that for any vertex $a \in A$ such that $k$ is connected to $a$, the edge $[k, a]$ is weighted 2 . Let us write

$$
\left\{a_{i}: k \text { is connected to } a_{i}\right\}=\left\{a_{i_{1}}, \ldots, a_{i_{r}}\right\}, r \geq 1,
$$

where $1 \leq i_{1}<i_{2}<\ldots<i_{r} \leq m$. 
Subsubcase 3.1.1. $r \geq 3$. Then the subdiagram with the vertices $\left\{a_{i_{1}}, k, a_{i_{r}}\right\}$ is of type $C_{n}^{(1)}$

Subsubcase 3.1.2. $\quad r=2$. If the vertices $a_{i_{1}}$ and $a_{i_{2}}$ are not connected to each other, then the subdiagram on vertices $\left\{a_{i_{1}}, k, a_{i_{2}}\right\}$ is of type $C_{n}^{(1)}$. Let us now assume that $a_{i_{1}}$ and $a_{i_{2}}$ are connected to each other. Then we have the following:

(i) If $i_{1} \neq 1$, then the subdiagram on vertices $\left\{a_{1}, b_{1}, k, a_{i_{2}}\right\}$ is of type $C_{n}^{(1)}$ (Fig 15).

(ii) If $i_{2} \neq m$ then the subdiagram on vertices $\left\{a_{m}, c_{1}, k, a_{i_{1}}\right\}$ is of type $C_{n}^{(1)}$.

(iii) If neither of the conditions in (i,ii) holds, then $i_{1}=1$ and $i_{2}=2=m$, so $\mu_{k}(\Gamma k)$ is of type $F_{4}^{(1)}\left(3^{2} ; 0\right)$.

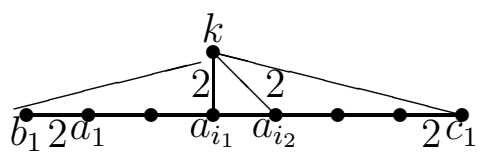

Figure 15: figure for Subsubcase 3.1.2

Subsubcase 3.1.3. $r=1$. Then we have the following:

(i) If $1<i_{1}<m$, then the subdiagram on vertices $\left\{k, a_{i_{1}}, a_{i_{1}-1}, a_{i_{1}+1}\right\}$ is of type $B_{n}^{(1)}$

(ii) Suppose that the condition of (i) does not hold. Then, without loss of generality, we may assume that $i_{1}=1$. If $m>2$, then the subdiagram on vertices $\left\{a_{1}, k, c_{1}, a_{m}\right\}$ is of type $C_{n}^{(1)}$. If $m=2$, then the diagram $\mu_{k}(\Gamma k\}$ is of type $F_{4}^{(1)}\left(3^{2} ; 0 ; 1^{1}\right)$. If $m=1$, then $\mu_{k}(\Gamma k\}$ is of type $B_{n}^{(1)}$.

Subcase 3.2. $\quad k$ is not connected to any vertex in $\left\{a_{1}, \ldots, a_{m}\right\}$. Then we have the following: if $m>2$, then the subdiagram on vertices $\left\{a_{1}, b_{1}, k, c_{1}, a_{m}\right\}$ is of type $C_{n}^{(1)}$; if $m=2$ then $\mu_{k}(\Gamma k)$ is of type $F_{4}^{(1)}\left(4^{1} ; 3^{1}\right)$; if $m=1$ then then $\mu_{k}(\Gamma k)$ is of type $B_{n}^{(1)}(r)$.

\subsection{Proof of Theorem 3.2}

In view of Theorem 3.1, the theorem is the same as Lemma 6.1, which we already proved.

Remark 7.1 By enlarging the set of representatives for mutation classes of minimal 2infinite diagrams, we obtain the following recognition criterion for 2-finite diagrams:

Using at most 9 mutations, any 2-infinite diagram can be transformed into a diagram which contains a either a diagram from Table 1 or one of the following extended Dynkin diagrams: $E_{6}^{(1)}, E_{7}^{(1)}, E_{8}^{(1)}, F_{4}^{(1)}$.

To prove this statement, one may note that any (minimal 2-infinite) diagram which is not in Table 1 has at most 9 vertices. We also note that it is enough to prove the theorem for any diagram $\Gamma$ (which is not in Table 1) from our list, because any 2-infinite diagram contains a subdiagram from our list (Theorem 3.1). This is manageable despite the relatively large number of diagrams in Tables 2-6. Although we checked this by hand we do not give the proof here. 


\section{The list of minimal 2-infinite diagrams}

Unless otherwise stated, any diagram in this list is assumed to have an arbitrary orientation which does not contain any non-oriented cycle.

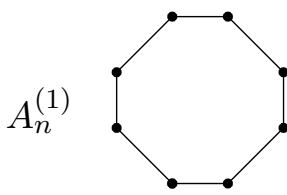

non-oriented
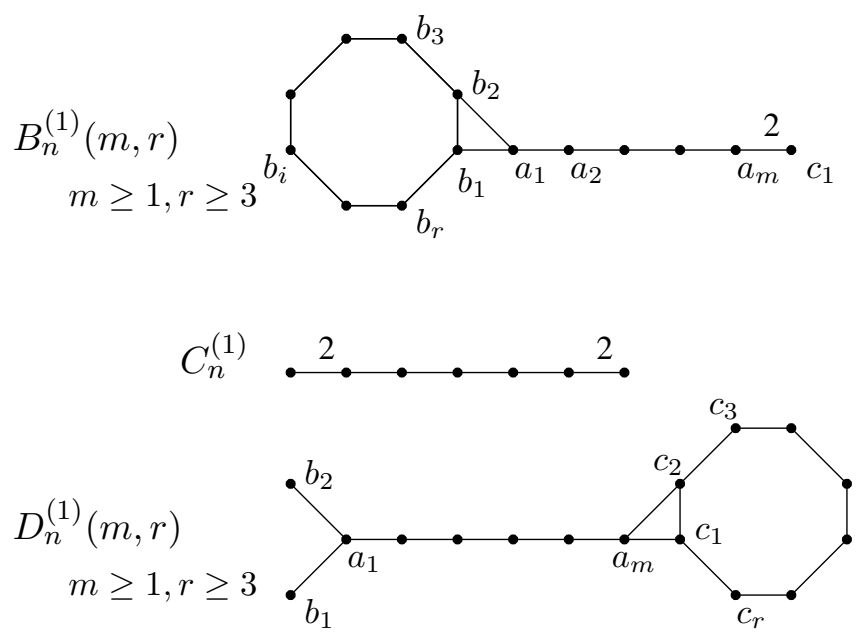

$D_{n}^{(1)}$
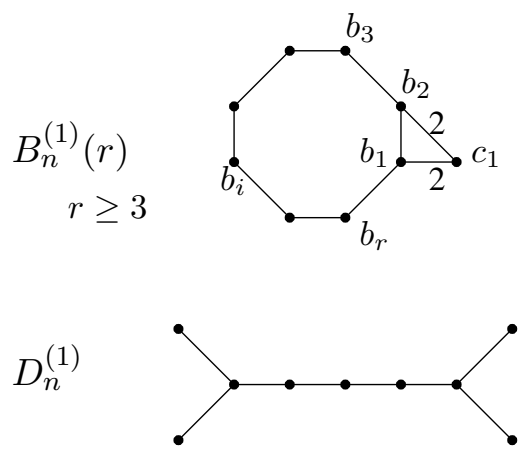

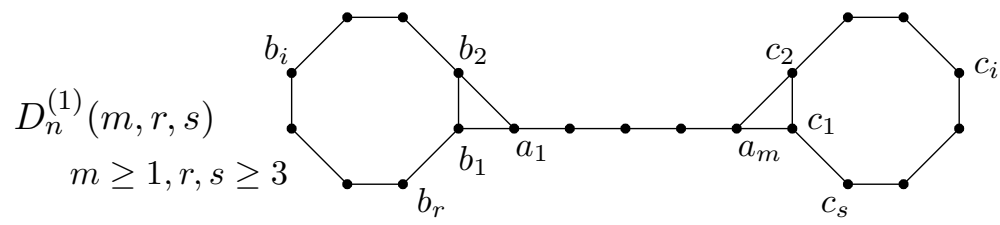

$$
\begin{aligned}
& D_{n}^{(1)}(r) \\
& \quad r \geq 3
\end{aligned}
$$
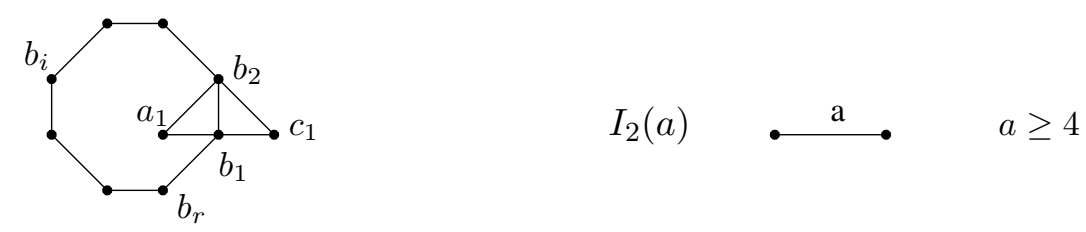

Table 1: Minimal 2-infinite diagrams that appear in series 


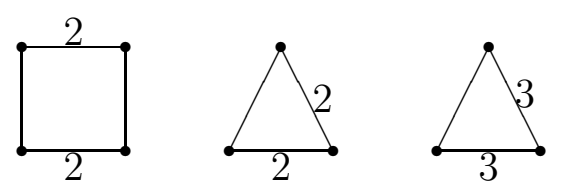

(each cycle is non-oriented)

Table 2: Minimal 2-infinite diagrams which are not mutation equivalent to any extended Dynkin diagram in Fig. 3.

In the following tables, we index the diagrams as follows. For any exceptional extended Dynkin diagram $X$, we denote by $X\left(c_{i}^{m_{i}} ; t ; b_{i}^{l_{i}} ; d_{i}^{n_{i}}\right)$ a minimal 2-infinite diagram which is mutation equivalent to $X$ and which has: $m_{i}$ cycles of length $c_{i}, t$ triangles which are not adjacent to any cycle, $l_{i}$ branches of length $l_{i}$ (here a branch is a maximal chain which is not adjacent to any cycle), $n_{i}$ vertices which are connected to precisely $d_{i}$ vertices.

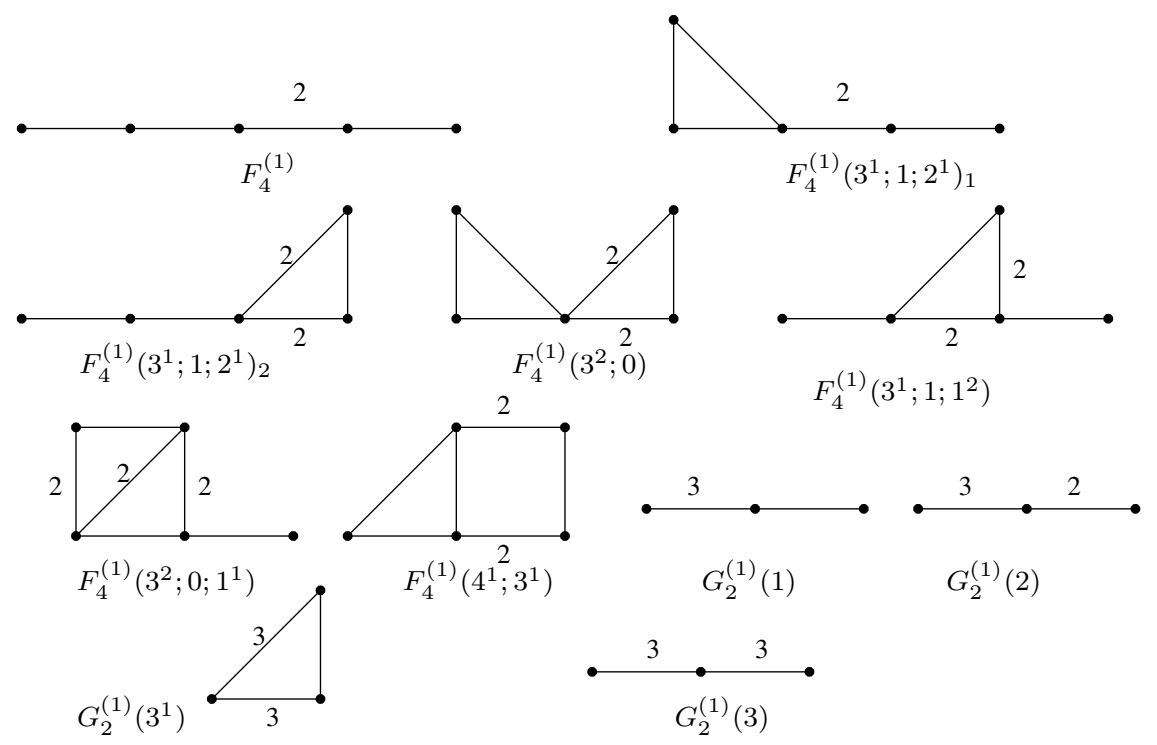

Table 3: Minimal 2-infinite diagrams which are mutation equivalent to $F_{4}^{(1)}$ or $G_{2}^{(1)}$.
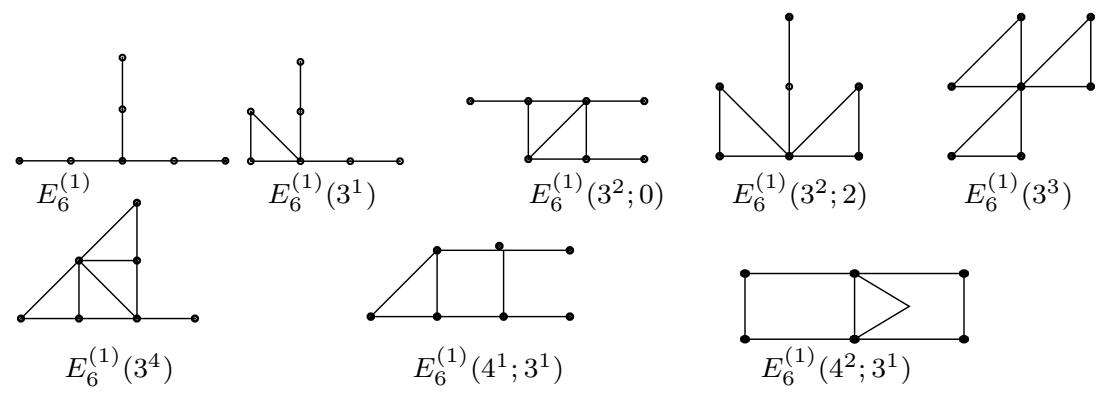

Table 4: Minimal 2-infinite diagrams which are mutation equivalent to $E_{6}^{(1)}$. 


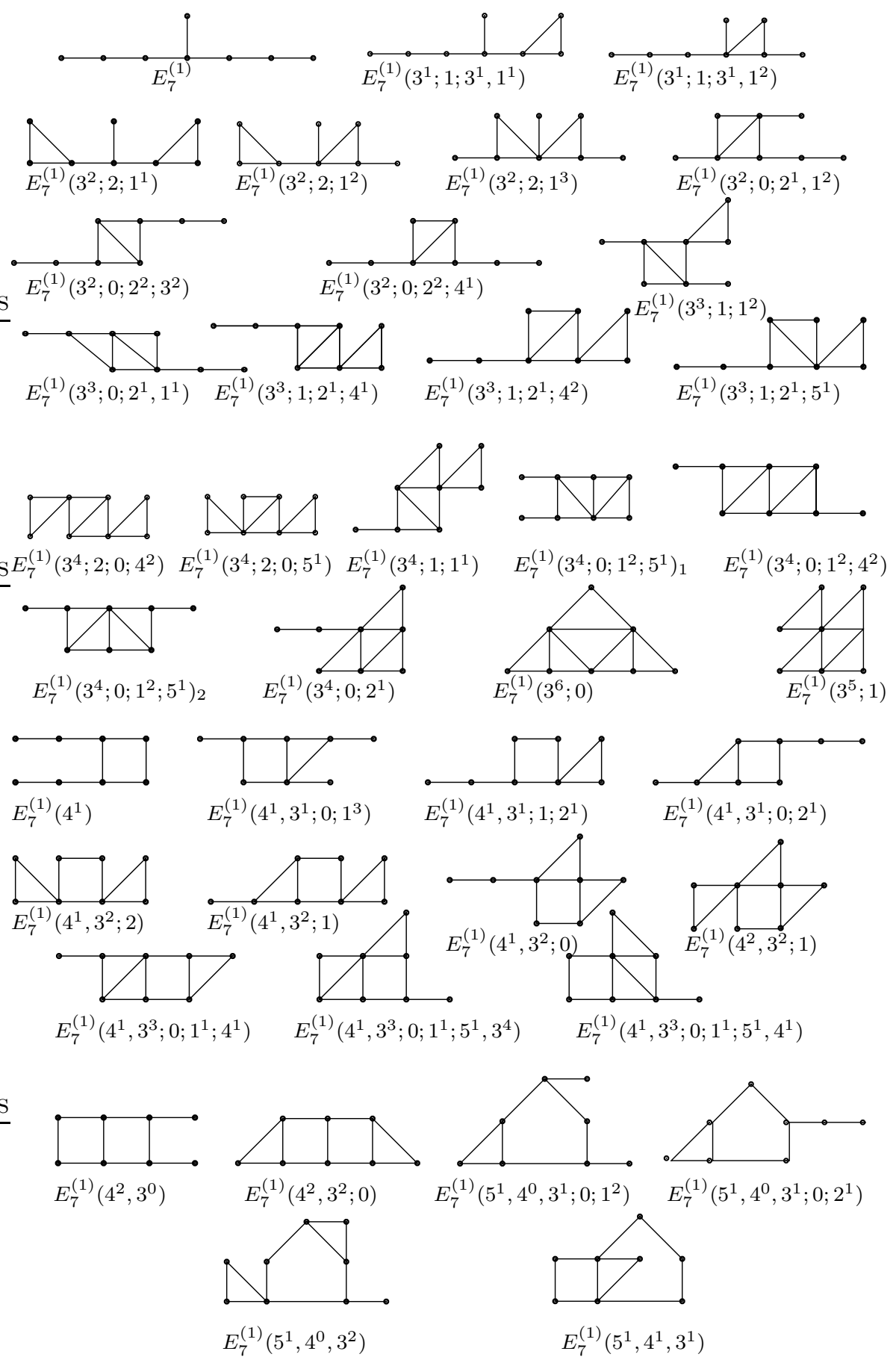

Table 5: Minimal 2-infinite diagrams which are mutation equivalent to $E_{7}^{(1)}$. 


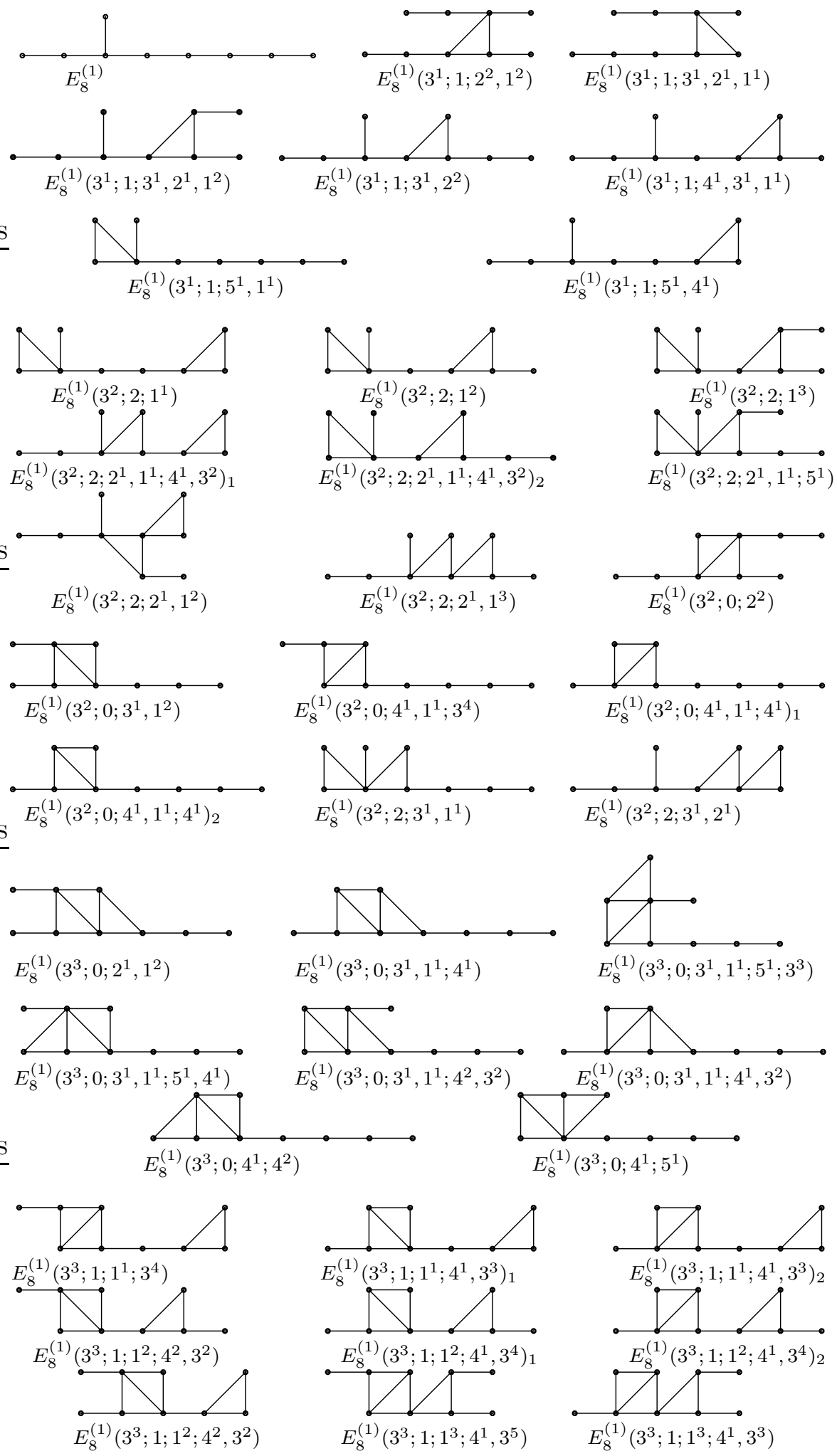

Table 6: Minimal 2-infinite diagrams which are mutation equivalent to $E_{8}^{(1)}$ 


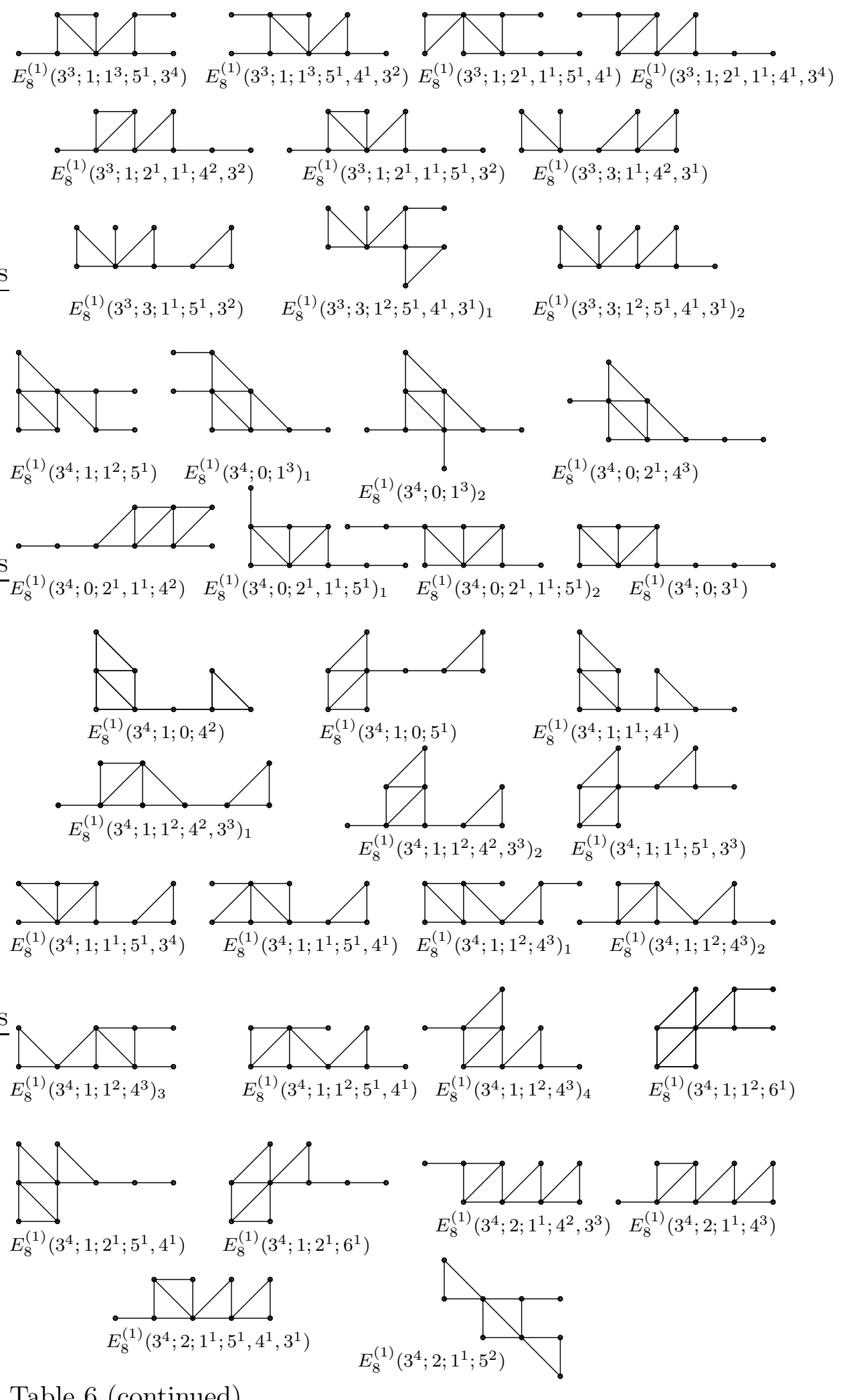

Table 6 (continued) 


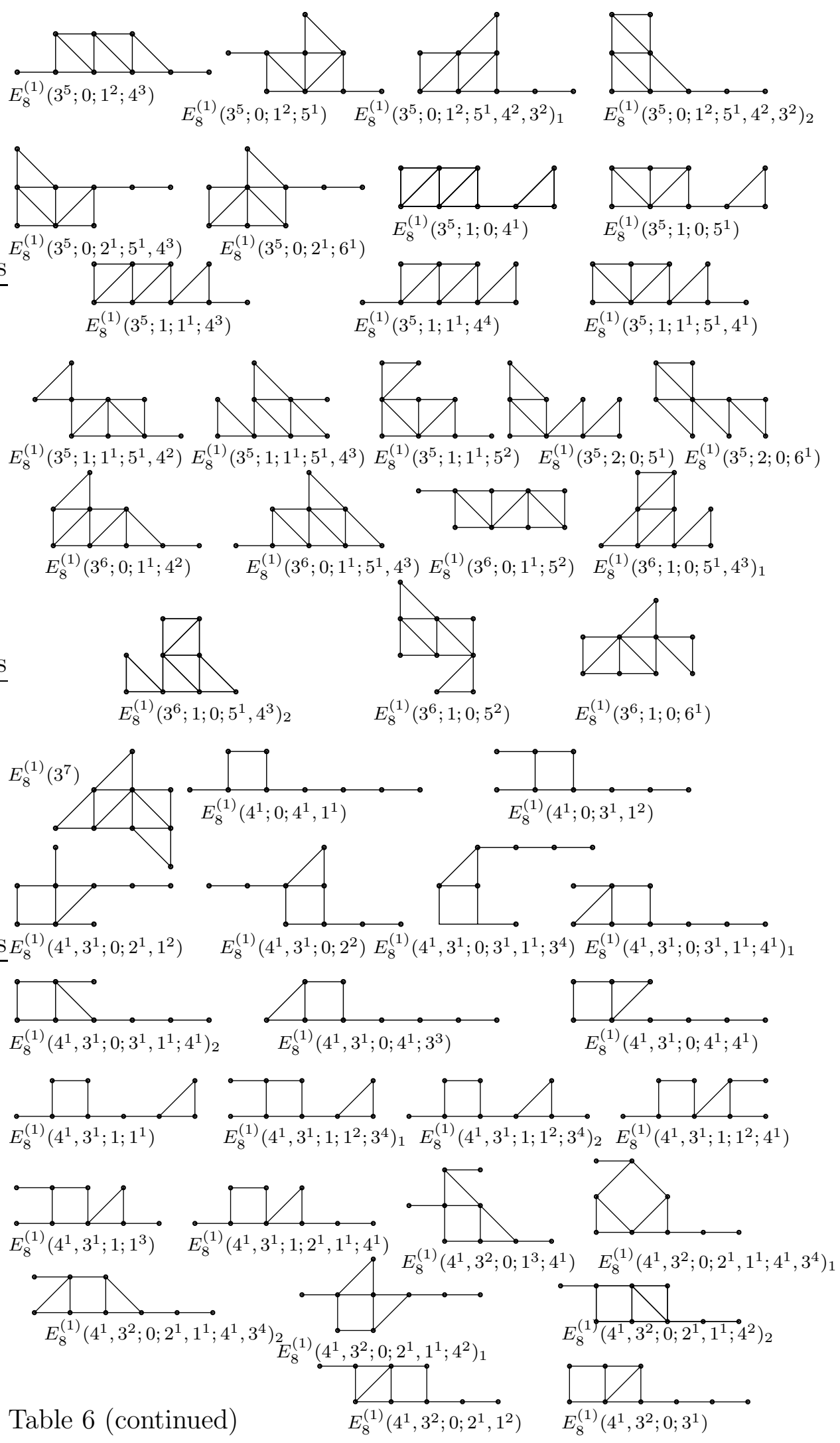

THE ELECTRONIC JOURNAL OF COMBINATORICS 14 (2007), \#R3 


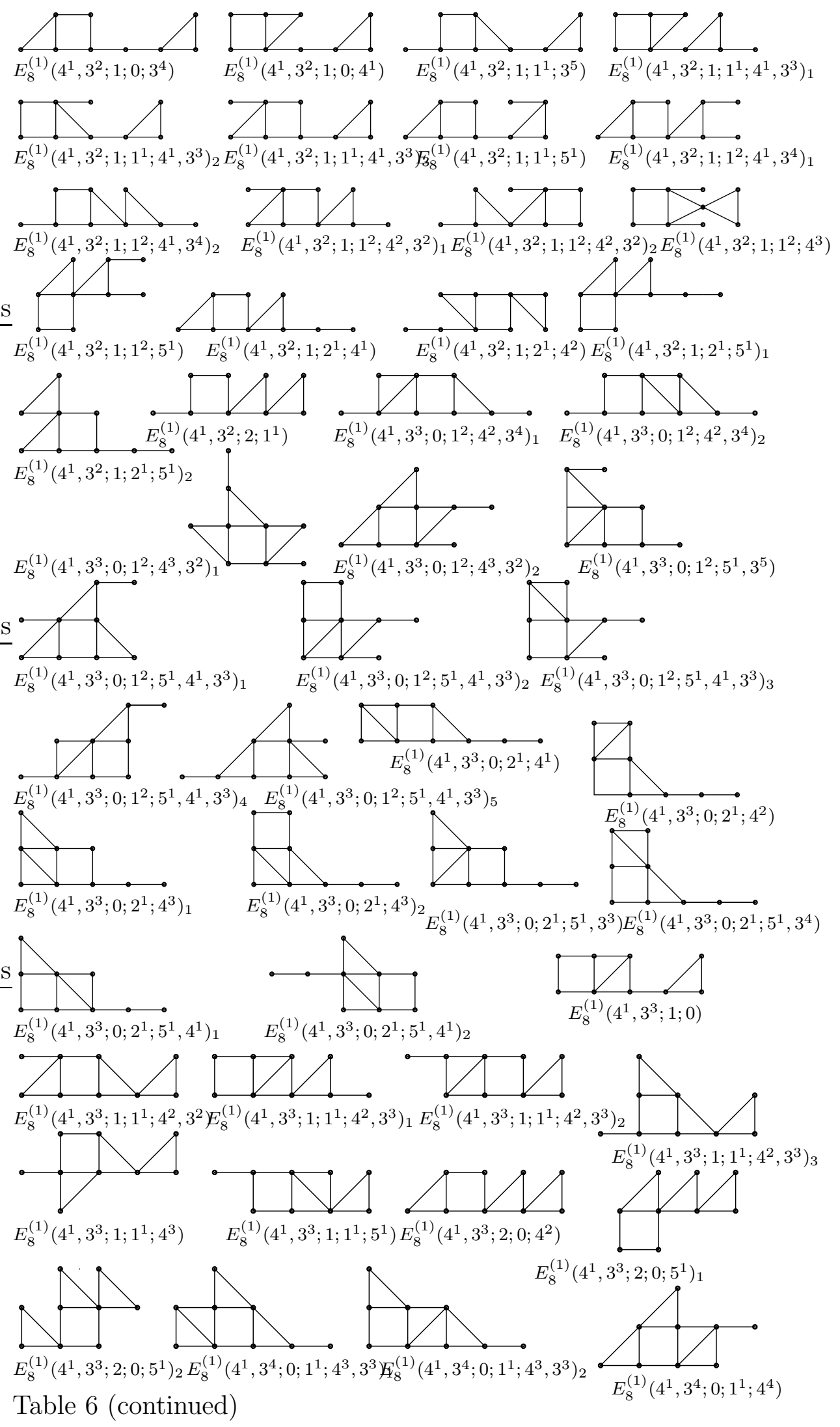

THE ELECTRONIC JOURNAL OF COMBINATORICS 14 (2007), \#R3 

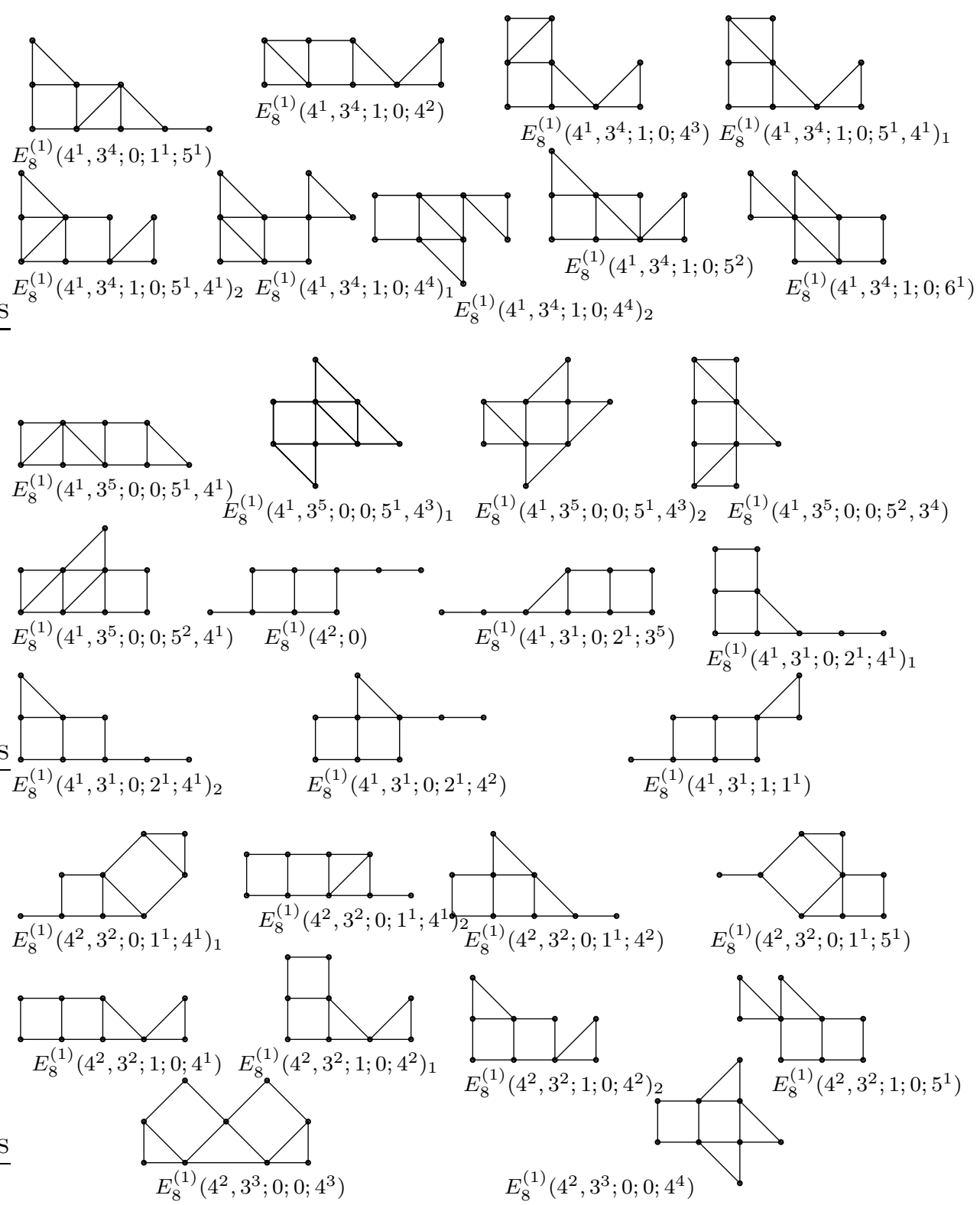

$E_{8}^{(1)}\left(4^{2}, 3^{3} ; 0 ; 0 ; 4^{4}\right)$
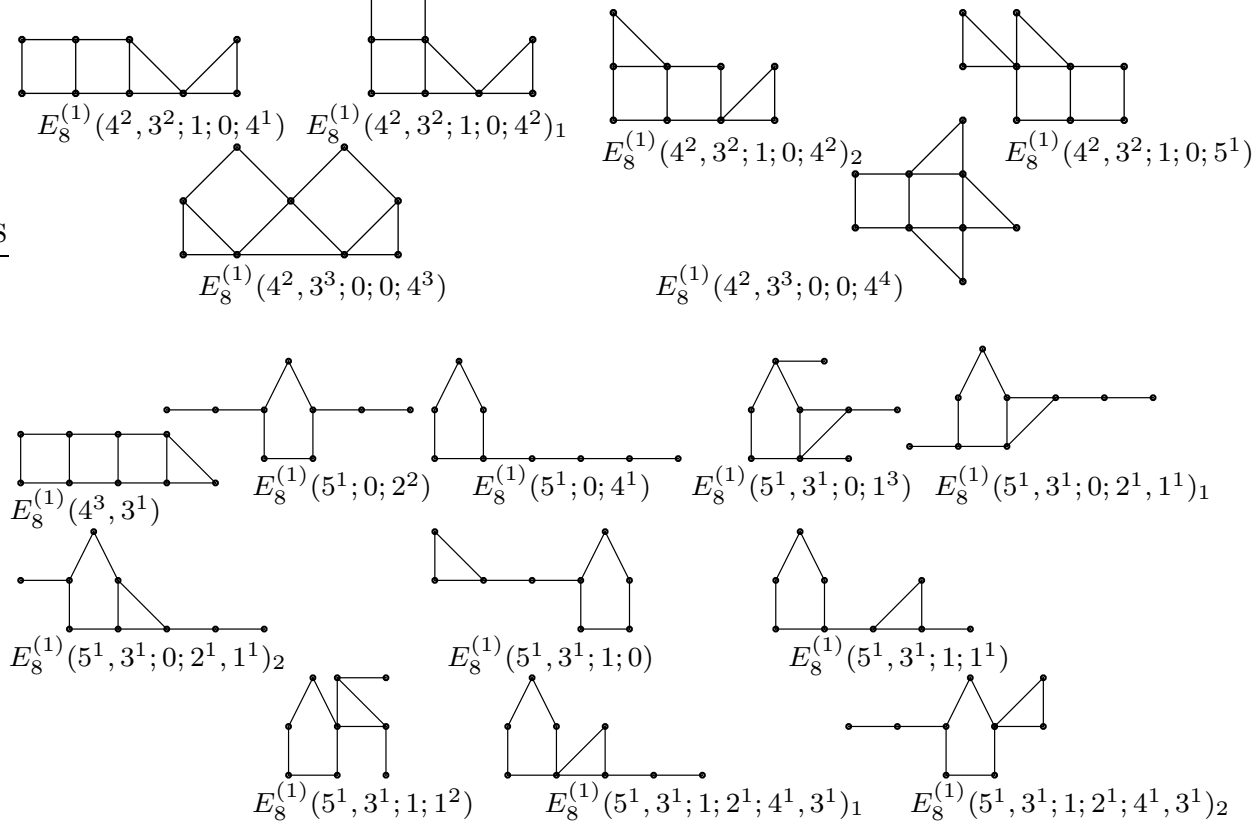

Table 6 (continued)
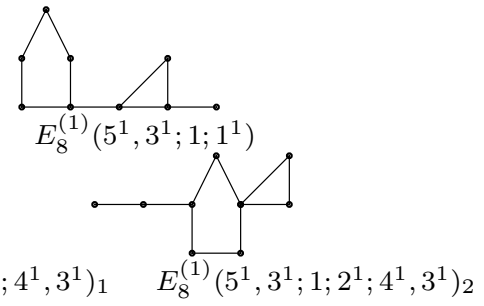

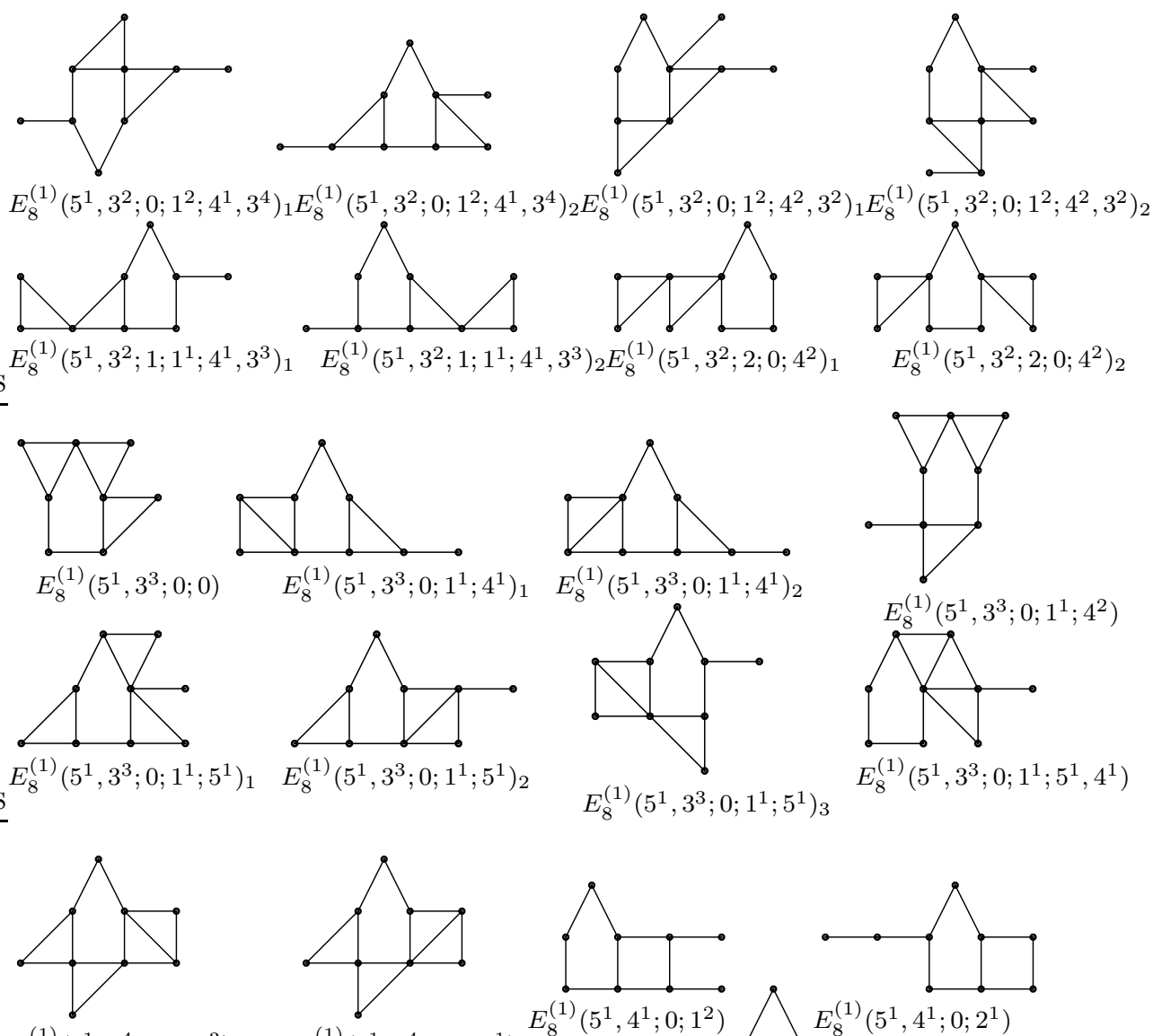

$E_{8}^{(1)}\left(5^{1}, 3^{4} ; 0 ; 0 ; 4^{3}\right)$
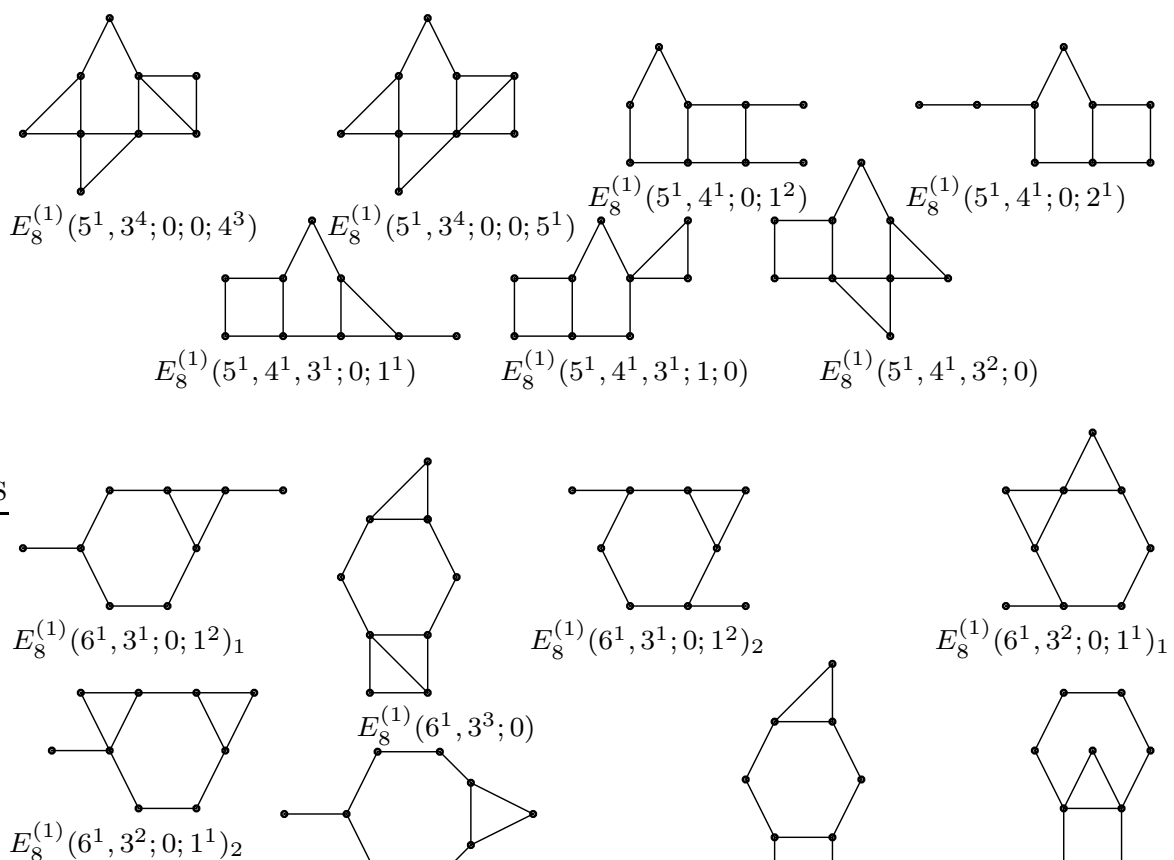

Table 6 (continued)

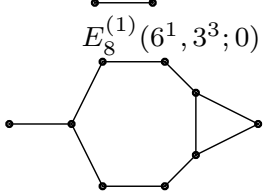

$E_{8}^{(1)}\left(7^{1}, 3^{1} ; 0\right) \quad E_{8}^{(1)}\left(6^{1}, 4^{1}, 3^{1} ; 0 ; 3^{4}\right)$

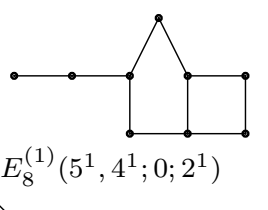




\section{References}

[1] A. Berenstein, S. Fomin and A. Zelevinsky, Cluster Algebras III, Upper bounds and double Bruhat cells. Duke Math. J. 126 (2005), no. 1, 1-52.

[2] R. Brown and S. Humphries, Orbits under symplectic transvections II: The case $K=F_{2}$, Proc. London Math. Soc. (3) 52 (1986), no. 3, 532-556.

[3] S. Fomin and A. Zelevinsky, The Laurent phenomenon, Adv. Applied Math. 28 (2002), no. $2,119-144$.

[4] S. Fomin and A. Zelevinsky, Cluster Algebras I, J. A. Math. Soc. 12 (2003), 335-380

[5] S. Fomin and A. Zelevinsky, Cluster Algebras II, Inv. Math. 12 (2003), 335-380

[6] M. Gekhtman, M. Shapiro, A. Vainshtein, Cluster algebras and Poisson Geometry, Moscow Math. Journal(2003), no. 3, 899-934, 1199.

[7] M. Gekhtman, M. Shapiro, A. Vainshtein, Cluster algebras and Weil-Petersson forms, Duke Math. J. 127 (2005), no. 2, 291-311.

[8] D. Happel, D. Vossieck, Minimal algebras of infinite representation type with preprojective component, Manuscripta Math. 42 (1983), no. 2-3, 221-243.

[9] W.A.M. Janssen, Skew-symmetric vanishing lattices and their monodromy groups, Math. Ann. 266 (1983), 115-133

[10] V. Kac, Infinite dimensional Lie algebras, Cambridge University Press (1991).

[11] A. Seven, Orbits of groups generated by transvections over $F_{2}$, J. Algebraic Combin. 21 (2005), no. 4, 449-474.

[12] A. Seven, Recognizing cluster algebras of finite type, ArXiv math.CO/0406545.

[13] A. Seven, Combinatorial aspects of double Bruhat cells and cluster algebras, Ph.D. thesis, Northeastern University, July 2004.

[14] B. Shapiro, M. Shapiro, A. Vainshtein and A. Zelevinsky, Simply-laced Coxeter groups and groups generated by symplectic transvections, Michigan Mathematical Journal, 48, 2000, 531-552.

[15] http://mystic.math.neu.edu/aseven 